\title{
A stabilisation approach for topology optimisation of hyperelastic structures with the SIMP method
}

\author{
R. Ortigosa ${ }^{\dagger 1}$, D. Ruiz ${ }^{\ddagger}$, A. J. Gil ${ }^{\amalg 2}$, A. Donoso ${ }^{\ddagger}$, J. C. Bellido ${ }^{\ddagger 3}$ \\ ${ }^{\dagger}$ Computational Mechanics 83 Scientific Computing Group, Technical University of Cartagena, \\ Campus Muralla del Mar, 30202 Cartagena (Murcia), Spain \\ ${ }^{\ddagger}$ Departamento de Matematicas, ETSII, Universidad de Castilla-La Mancha, 13071 Ciudad Real, Spain \\ ${ }^{\amalg}$ Zienkiewicz Centre for Computational Engineering, College of Engineering \\ Swansea University, Bay Campus, SA1 8EN, United Kingdom
}

\begin{abstract}
This paper presents a novel computational approach for SIMP-based Topology Optimisation (TO) of hyperelastic materials at large strains. During the TO process for structures subjected to very large deformations, and especially in the presence of intermediate density regions, the standard Newtonsolver (or its arc length variant) have been reported not to converge (refer to References [15, 27, 33]). In this paper, the new TO stabilisation technique proposed in [1] in the context of level-set TO, initially devised to alleviate numerical instabilities inherent to level-set TO, is extended for the TO by means of the SIMP method. The success of the methodology rests on the combination of two distinct key ingredients. First, the nonlinear equilibrium equations of motion for intermediate TO design stages are solved in a non-exact albeit consistent incrementally linearised fashion by splitting the design load into a number of load increments. Second, the resulting linearised tangent elasticity tensor is locally stabilised (regularised) in order to prevent its loss of positive definiteness and, thus, avoid the loss of convexity of the discrete tangent operator. This solution strategy is shown to be extremely robust in the context of density-based TO, where the constitutive law of the underlying evolving solid structure is a mixture of solid and void constituents, the latter classically defined by means of a fictitious strain energy. The robustness and applicability of this TO methodological approach are thoroughly demonstrated through an ample spectrum of challenging numerical examples, ranging from benchmark two-dimensional (plane stress) examples to larger scale three-dimensional applications. Crucially, the performance of all the final designs has been tested at a post-processing stage without adding any source of artificial stiffness. Specifically, an arc-length Newton-Raphson method has been employed in conjunction with a ratio of the material parameters for void and solid regions of $10^{-12}$.
\end{abstract}

Keywords: Topology optimisation, SIMP method, nonlinear elasticity, policonvexity

\footnotetext{
${ }^{1}$ Corresponding author: rogelio.ortigosa@upct.es

${ }^{2}$ Corresponding author: a.j.gil@swansea.ac.uk

${ }^{3}$ Corresponding author: JoseCarlos.Bellido@uclm.es
} 


\section{Introduction}

Since the pioneering work of Bendsøe and Kikuchi [2], the scientific field of structural Topology Optimisation (TO) has been extraordinarily prolific. According to Sigmund and Maute [3], TO methods can be broadly classified into: density-based methods, with the Solid Isotropic Material with Penalisation (SIMP) method as their maximum representative [4, 5], level-set methods [6, 7], phase-field methods [8], topological derivative methods [9] and evolutionary methods [10].

Despite the maturity and success of structural TO methods in the linear (small displacements and strains) elastic regime, this is not the case when applied to hyperelastic structures undergoing large displacements and/or strains. In these scenarios, the inevitable development of geometric non-linearities leads to the onset of numerical instabilities which can seriously hinder the robustness of TO methods. Specifically, (i) the loss of convexity of the invariant-based representation of the strain energy, which ultimately yields loss of positive definiteness of the tangent operator and (ii) the presence of low and intermediate density regions (in the case of the SIMP method [11]) or the nucleation process that occurs when initiating the algorithm from a generic seed (in the case of the level-set method [12]). The combination of above two factors poses a serious limitation to the robust application of well-established nonlinear solution techniques, such as the arc-length method [13], despite the latter being originally designed to surpass limit points and track snap-through and snap-back equilibrium path types.

In the SIMP method, the (hyperelastic) strain energy density of the material consists of a convex combination of the strain energy density of the solid and void phases $[14,15]$. In the limit case, when the design density field tends to one (or zero), the convex energy combination degenerates to the energy of the solid (or void) phase. Intermediate density regions (therefore consisting of a mixture of both phases) are reported to be the most prone to exhibit instabilities $[11,16]$. The underlying reason lies in the physical constraints that the strain energy density of the solid phase must comply with which, unavoidably, degrades the stability of the tangent operator of the intermediate density regions.

The energy density of the solid phase must represent a physically and mathematically admissible constitutive law (in contrast to that of the fictitious void phase). As an example, the strain energy density must be objective or frame indifferent [13] and, moreover, if the material is isotropic, the RivlinEricksen representation theorem establishes that the strain energy density must be expressed in terms of the invariants of the deformation gradient tensor $\boldsymbol{F}$, its cofactor $\boldsymbol{H}$ or its Jacobian $J[17,18]$. Further convexity-type restrictions must also be incorporated into the strain energy of the solid phase to ensure that the equilibrium equations are well-posed and that their numerical (i.e. Finite Element) solution is devoid from parasitic mesh dependence and strongly localised deformations [19]. Although one might be tempted to employ a convex (in $\boldsymbol{F}$ ) strain energy density for the solid phase, in order to circumvent altogether the stability issues previously mentioned, this would not only prevent the capturing of physical (realistic) buckling phenomena but it would also violate the principle of material frame indifference [18]. As a result, the so-called rank-one (or ellipticity) local condition is typically preferred [19-21]. With this in mind, a sufficient condition which guarantees the rank-one convexity condition and further physical requirements (material frame indifference, possibility of capturing buckling ...) is that of polyconvexity $[20,22]$, which requires the strain energy functional to be written as a convex multi-variable function of $\{\boldsymbol{F}, \boldsymbol{H}, J\}$.

Several authors have ventured in the field of structural TO at large displacements/strains by using either polyconvex constitutive models or the Saint Venant-Kirchhoff model ${ }^{4}[12,15,24-32]$, where some

\footnotetext{
${ }^{4}$ The Saint Venant Kirchhoff model is non-elliptic and hence it is not recommended except for materials experiencing
} 
strategies have been put forward to overcome some of the instabilities associated with intermediate density regions. For instance, the additive hyperelasticity technique presented in [33]; the combination of a polyconvex strain energy density in conjunction with an ad-hoc relaxation introduced in [27] to stabilise those excessively distorted elements of an underlying Finite Element mesh, or an original interpolation scheme for the strain energy density as proposed in [15]. We also mention the very recent work [34] on the optimal design of hyperelastic structures in the context of thermoelasticity.

Very recently, some of the authors of this manuscript have put forward in [1] a novel computational approach for the level-set based TO of hyperelastic materials at large strains. The regularisation technique proposed therein combines two key features: (i) the relaxed (non-exact) solution of the equilibrium equations in a consistently linearised incremental fashion and (ii) the localised regularisation of the elasticity tensor preventing its loss of positive definiteness and, hence, that of the tangent operator. Inspired by the success of this approach, we explore in this paper this novel methodology and carefully adapt it in the context of SIMP-based TO, where the source of numerical instabilities is of a very different nature.

It is important to make a clarifying remark regarding the possible choice of objective function in the context of non-linear elasticity. It is well-known that in the context of linearised elasticity, maximizing stiffness is equivalent to minimizing the end-compliance (or virtual work of the external loads). In fact, in this context, end-compliance minimisation is also equivalent to minimising the strain energy or its complementary one. This equivalence does not extend to the non-linear regime. In fact, as reported by $[30,32]$, the end-compliance might not be the best measure to characterise stiffness in this regime, and instead, they advocate for the minimisation of the complementary work. However, the scope of this paper is not on the suitability of the objective function in order to characterise the stiffness of the structure, but rather, on presenting a new methodology that can address the numerical difficulties in TO by means of the SIMP method, which manifest regardless of the choice of objective function.

The paper is organised in the following Sections: Section 2 briefly recalls some fundamentals of Nonlinear Solid Mechanics. Section 3 describes succinctly the minimisation setting typically used in nonlinear hyperelasticity. Section 4 presents the incrementally linearised regularised TO methodology and puts forward two new TO solution strategies. Finally, Section 5 shows a series of numerical examples in order to illustrate the robustness and applicability of the methodology. Section 6 provides some concluding remarks.

\section{3D Nonlinear continuum mechanics}

\subsection{Kinematics}

Let us consider the motion of a continuum with reference or material configuration $\Omega_{0} \subset \mathbb{R}^{d}(d=$ $\{2,3\}$ ) and boundary $\partial \Omega_{0}$ with outward unit normal $\boldsymbol{N}$. After the motion, the continuum occupies a deformed or spatial configuration $\Omega \subset \mathbb{R}^{d}$ and boundary $\partial \Omega$ with outward unit normal $\boldsymbol{n}$ (see Figure 1). A mapping $\phi$ links a material particle from the reference configuration $\boldsymbol{X} \in \Omega_{0}$ to the deformed configuration $\boldsymbol{x} \in \Omega$ according to $\boldsymbol{x}=\boldsymbol{\phi}(\boldsymbol{X})=\boldsymbol{X}+\boldsymbol{u}(\boldsymbol{X})$, with $\boldsymbol{u}$ the displacement field. Associated with $\boldsymbol{\phi}$, it is possible to define the two-point ${ }^{5}$ deformation gradient tensor $\boldsymbol{F}$ as

$$
\boldsymbol{F}=\nabla_{0} \boldsymbol{\phi}(\boldsymbol{X})=\boldsymbol{I}+\boldsymbol{\nabla}_{0} \boldsymbol{u}(\boldsymbol{X}) ; \quad F_{i I}=\frac{\partial \phi_{i}}{\partial X_{I}}
$$

very moderate strains [23].

${ }^{5}$ Lowercase (uppercase) indices are used to refer to the spatial (material) configuration. 
where $\boldsymbol{\nabla}_{0}(\bullet)=\frac{\partial(\bullet)}{\partial \boldsymbol{X}}$ represents the material gradient operator and $\boldsymbol{I}$ the second-order identity tensor. For completeness, it is also useful to introduce two additional kinematic measures, namely the co-factor $\boldsymbol{H}$ and the Jacobian $J$ of the deformation, defined as

$$
J=\operatorname{det} \boldsymbol{F} ; \quad \boldsymbol{H}=J \boldsymbol{F}^{-T} .
$$

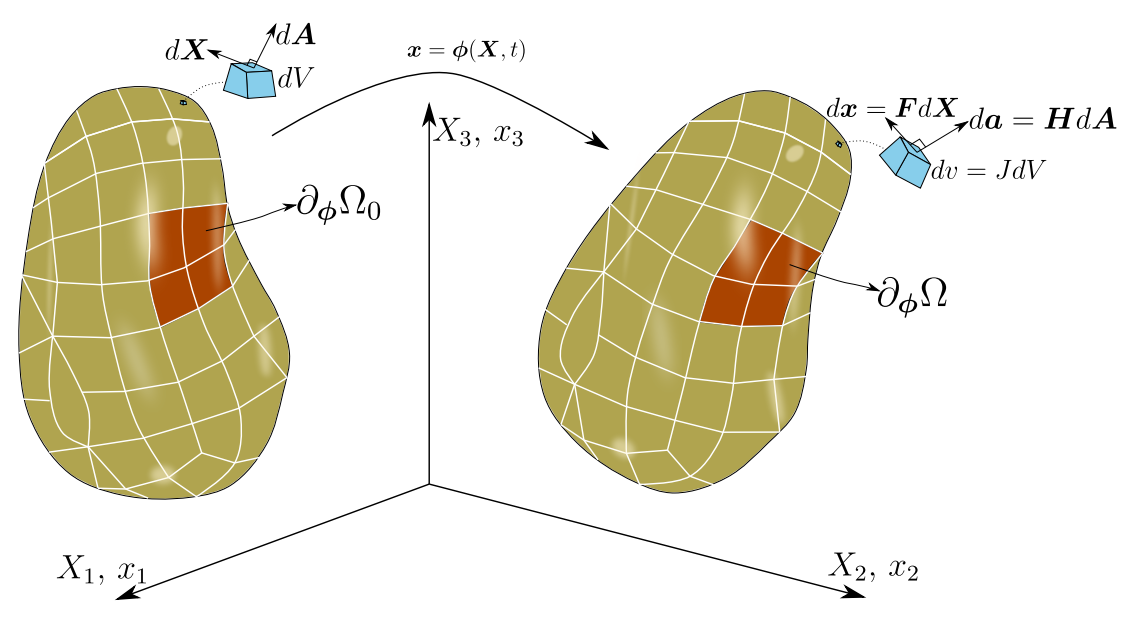

Figure 1: Strain measures in 3D: the deformation gradient tensor $\boldsymbol{F}$, its co-factor $\boldsymbol{H}$ and its Jacobian $J$.

Alternative, yet equivalent expressions for $\boldsymbol{H}$ and $J$ are $[35,36]$

$$
\boldsymbol{H}=\frac{1}{2} \boldsymbol{F} \times \boldsymbol{F} ; \quad J=\frac{1}{3} \boldsymbol{H}: \boldsymbol{F},
$$

where the tensor cross product operation $\times$ between two second-order tensors $\boldsymbol{A} \in \mathbb{R}^{3 \times 3}$ and $\boldsymbol{B} \in \mathbb{R}^{3 \times 3}$ reads as $(\boldsymbol{A} \times \boldsymbol{B})_{i I}=\mathcal{E}_{i j k} \mathcal{E}_{I J K} A_{j J} B_{k K}$ and $\mathcal{E}_{i j k}\left(\mathcal{E}_{I J K}\right)$ represents the third order alternating tensor.

\subsection{Governing equations in nonlinear continuum mechanics}

The partial differential equations and associated boundary conditions governing the motion of the continuum $\Omega_{0}$ described in Section 2.1 can be written under a Lagrangian formalism as

$$
\begin{aligned}
\mathrm{DIV} \boldsymbol{P}+\boldsymbol{f}_{0} & =\mathbf{0} ; & & \text { in } \Omega_{0} ; \\
\boldsymbol{P} \boldsymbol{N} & =\boldsymbol{t}_{0} ; & & \text { on } \partial_{N} \Omega_{0} ; \\
\boldsymbol{\phi} & =\overline{\boldsymbol{\phi}} ; & & \text { on } \partial_{\boldsymbol{\phi}} \Omega_{0},
\end{aligned}
$$

where $(4)_{a}$ represents the classical equilibrium equations, $\boldsymbol{f}_{0}$ denotes the body force per unit undeformed volume $\Omega_{0}$ and $\boldsymbol{t}_{0}$ the traction force per unit undeformed area applied on the Neumann boundary $\partial_{N} \Omega_{0} \subset \partial \Omega_{0}$. Furthermore, $\partial_{\phi} \Omega_{0}$ represents the portion of the boundary $\partial \Omega_{0}$ where Dirichlet boundary conditions are applied on $\boldsymbol{\phi}$, with $\partial_{N} \Omega_{0} \cup \partial_{\phi} \Omega_{0}=\partial \Omega_{0}$ and $\partial_{N} \Omega_{0} \cap \partial_{\phi} \Omega_{0}=\emptyset$. In addition, $\boldsymbol{P}$ represents the first Piola-Kirchhoff stress tensor and the local conservation of angular momentum leads to the wellknown tensor condition $\boldsymbol{P} \boldsymbol{F}^{T}=\boldsymbol{F} \boldsymbol{P}^{T}$. Finally, DIV $(\bullet)$ represents the material divergence operator, i.e. $(\operatorname{DIV} \boldsymbol{P})_{i}=\frac{\partial P_{i I}}{\partial X_{I}}$ 


\subsection{Polyconvex hyperelasticity}

In order to close the system of equilibrium (state) equations in (4), a constitutive law relating the first Piola-Kirchhoff stress tensor $\boldsymbol{P}$ and the deformation gradient tensor $\boldsymbol{\nabla}_{0} \boldsymbol{\phi}$ is needed. In the case of reversible elasticity, this is achieved through the introduction of a strain energy density $e$ per unit undeformed volume typically written as $e=e\left(\boldsymbol{X}, \nabla_{0} \phi\right)$. We consider in this work polyconvex strain energy density functionals of the form [20]

$$
e\left(\boldsymbol{X}, \boldsymbol{\nabla}_{0} \phi\right)=W(\boldsymbol{X}, \mathcal{V}) ; \quad \mathcal{V}=\{\boldsymbol{F}, \boldsymbol{H}, J\}
$$

where $W$ is a convex function with respect to the extended set $\mathcal{V}$ of kinematic measures. Notice that the dependence of $e$ (and $W$ ) in (5) with respect to the material position $\boldsymbol{X}$ indicates that the material properties can potentially vary with the material position. In the simpler case where the material parameters are not spatially dependant (i.e. $e=e\left(\boldsymbol{\nabla}_{0} \boldsymbol{\phi}\right)$ ), a commonly used polyconvex constitutive law is given by the so-called Mooney-Rivlin model, defined as

$$
e\left(\nabla_{0} \phi\right)=W(\mathcal{V}):=\frac{\mu_{1}}{2} I I_{\boldsymbol{F}}+\frac{\mu_{2}}{2} I I_{\boldsymbol{H}}+f(J) ; \quad f(J)=\frac{\lambda}{2}(J-1)^{2}-\left(\mu_{1}+2 \mu_{2}\right) \ln (J)-\frac{3}{2}\left(\mu_{1}+\mu_{2}\right),
$$

with $I I_{\boldsymbol{A}}=\boldsymbol{A}: \boldsymbol{A}=A_{i I} A_{i I}$ and where the material parameters $\mu_{1}, \mu_{2}, \lambda$ are related to the Youngs modulus $E$ and Poisson ratio $\nu$ as

$$
\mu_{1}+\mu_{2}=\frac{E}{2(1+\nu)} ; \quad \lambda-2\left(\mu_{1}+\mu_{2}\right)=\frac{E \nu}{(1+\nu)(1-2 \nu)} .
$$

Through the use of the directional derivatives of the strain energy density with respect to virtual and incremental variations of the mapping $\phi(\delta \phi$ and $\Delta \phi$, respectively) [13], the first Piola-Kirchhoff stress tensor $\boldsymbol{P}$ and the fourth order elasticity tensor $\mathcal{C}$ are obtained as

$$
\boldsymbol{P}=\partial_{\nabla_{0} \phi} e\left(\boldsymbol{X}, \nabla_{0} \phi\right) ; \quad \mathcal{C}=\partial_{\nabla_{0} \phi \nabla_{0} \phi}^{2} e\left(\boldsymbol{X}, \nabla_{0} \phi\right) .
$$

Remark 1. Alternative expressions for the first Piola-Kirchoff stress tensor $\boldsymbol{P}$ and the elasticity tensor $\mathcal{C}$ can be obtained when considering the extended representation of the strain energy density, namely $W(\boldsymbol{X}, \mathcal{V})$ (or $W(\mathcal{V})$ for a homogeneous material). Indeed, as shown in Bonet et al. [23, 35], the stress tensor $\boldsymbol{P}$ can also be expressed as

$$
\boldsymbol{P}=\partial_{\boldsymbol{F}} W+\partial_{\boldsymbol{H}} W \mathbf{\times} \boldsymbol{F}+\partial_{J} W \boldsymbol{H},
$$

and the elasticity tensor $\mathcal{C}$ as

$$
\begin{aligned}
\mathcal{C} & =\partial_{\boldsymbol{F} \boldsymbol{F}}^{2} W+\boldsymbol{F} \times \partial_{\boldsymbol{H}}^{2} W \times \mathbf{F}+\partial_{J J}^{2} W \boldsymbol{H} \otimes \boldsymbol{H}+\partial_{\boldsymbol{F} \boldsymbol{H}}^{2} W \times \boldsymbol{F}+\boldsymbol{F} \times \partial_{\boldsymbol{H} \boldsymbol{F}}^{2} W \\
& +\partial_{\boldsymbol{F} J}^{2} W \otimes \boldsymbol{H}+\boldsymbol{H} \otimes \partial_{J \boldsymbol{F}}^{2} W+\boldsymbol{F} \times \partial_{\boldsymbol{H} J}^{2} W \otimes \boldsymbol{H}+\boldsymbol{H} \otimes \partial_{J \boldsymbol{H}}^{2} W \times \boldsymbol{F} \\
& +\boldsymbol{I} \times\left(\partial_{\boldsymbol{H}} W+\partial_{J} W \boldsymbol{F}\right),
\end{aligned}
$$

with

$$
[\mathcal{I}]_{i I j J}=\delta_{i j} \delta_{i J} ; \quad[\mathcal{A} \times \boldsymbol{A}]_{i I j J}=\mathcal{A}_{i I p P} \mathcal{E}_{j p q} \mathcal{E}_{J P Q} A_{q Q} ; \quad[\boldsymbol{A} \times \mathcal{A}]_{i I j J}=\mathcal{E}_{i p q} \mathcal{E}_{I P Q} A_{p P} \mathcal{A}_{q Q j J}
$$

for any $\mathcal{A} \in \mathbb{R}^{3 \times 3 \times 3 \times 3}$ and $\boldsymbol{A} \in \mathbb{R}^{3 \times 3}$ and $\delta_{i j}$ denotes the $i j$-th component of the Kronecker delta tensor. The reader is referred to Ortigosa et al. [23] for a detailed derivation of $\mathcal{C}$ in (10). 


\section{Minimum structural compliance via SIMP approach}

In this work, we seek to obtain the optimum design of a structure via minimisation of its endcompliance $\mathcal{J}$ for a given load configuration, subjected to a prescribed volume fraction constraint $c$. The functional associated with the compliance can be formulated as

$$
\mathcal{J}(\boldsymbol{\phi})=\int_{\Omega_{0}} \boldsymbol{f}_{0} \cdot \boldsymbol{\phi} d V+\int_{\partial_{N} \Omega_{0}} \boldsymbol{t}_{0} \cdot \boldsymbol{\phi} d A,
$$

with $\boldsymbol{f}_{0}$ and $\boldsymbol{t}_{0}$ defined in (4) and where the mapping $\boldsymbol{\phi}(1)$ is restricted to the satisfaction of the (state) equilibrium equations (4) complemented by a given constitutive law. As it is usual in this kind of problems, $\phi$ implicitly depends on the density parameter through the constitutive law. In our case, the constitutive law is defined through a strain energy density formulated as a convex combination of solid and void regions as

$$
e\left(\boldsymbol{X}, \boldsymbol{\nabla}_{0} \boldsymbol{\phi}\right)=e^{\chi}\left(\chi(\boldsymbol{X}), \boldsymbol{\nabla}_{0} \boldsymbol{\phi}\right):=\chi(\boldsymbol{X}) e^{\text {solid }}\left(\boldsymbol{\nabla}_{0} \boldsymbol{\phi}\right)+[1-\chi(\boldsymbol{X})] e^{\text {void }}\left(\boldsymbol{\nabla}_{0} \boldsymbol{\phi}\right),
$$

where the design discrete field $\chi(\boldsymbol{X}) \in\{0,1\}$ represents a discrete scalar field (i.e. characteristic function) introduced in order to differentiate solid from void regions. The strain energy density of the solid region $e^{\text {solid }}$ can be defined through a polyconvex strain energy density as in (6), whilst the void region is typically [11] represented via a strain energy density $e^{\text {void }}$ formulated as

$$
e^{\mathrm{void}}\left(\boldsymbol{\nabla}_{0} \boldsymbol{\phi}\right):=\varepsilon e^{\operatorname{lin}}\left(\boldsymbol{\nabla}_{0} \boldsymbol{\phi}\right) ; \quad e^{\operatorname{lin}}\left(\boldsymbol{\nabla}_{0} \boldsymbol{\phi}\right):=\frac{1}{2}\left(\boldsymbol{\nabla}_{0} \boldsymbol{\phi}-\boldsymbol{I}\right):\left.\mathcal{C}\right|_{0}:\left(\boldsymbol{\nabla}_{0} \boldsymbol{\phi}-\boldsymbol{I}\right),
$$

where $\varepsilon$ is a dimensionless coefficient typically of the order of $10^{-6}$ to $10^{-5}$ and $\left.\mathcal{C}\right|_{0}$ is a fourth order linear elasticity tensor (normally obtained from evaluating the elasticity tensor of the solid region in the origin, i.e. $\left.\nabla_{0} \phi=I\right)$. Notice that, provided that $e^{\text {solid }}$ is polyconvex, since $e^{\text {void }}$ is convex, the resulting interpolated energy $e(13)$ is also polyconvex. The optimisation problem is closed through the consideration of the following volume constraint $g_{\Omega_{0}}(\chi)$

$$
g_{\Omega_{0}}(\chi):=\int_{\Omega_{0}} \chi(\boldsymbol{X}) d V-c\left|\Omega_{0}\right| \leq 0
$$

where $c$ is the volume fraction and $\left|\Omega_{0}\right|$ the total volume of the background optimisation domain. Unfortunately, a characteristic function-type formulation of the problem is not feasible because of its prohibitively high computational cost. Instead, the characteristic function $\chi(\boldsymbol{X})$ (with discrete values $\{0,1\})$ is replaced by an alternative design density field $\rho(\boldsymbol{X})$ taking values within the continuous interval $[0,1]$. Furthermore, in order to minimise the presence of grey ${ }^{6}$ values, we advocate in this paper for the use of the well-known Solid Isotropic Material with Penalisation (SIMP) method (see [14] and references therein). Furthermore, with the aim of circumventing mesh-dependence and chequerboard modes, we use a well-established filtering technique [11], which can be mathematically stated as the convolution product

$$
\tilde{\rho}(\boldsymbol{X})=(\rho * K)(\boldsymbol{X})=\int_{\Omega_{0}} \rho\left(\boldsymbol{X}^{\prime}\right) K\left(\left\|\boldsymbol{X}-\boldsymbol{X}^{\prime}\right\|\right) d V, \quad \boldsymbol{X}, \boldsymbol{X}^{\prime} \in \Omega_{0},
$$

\footnotetext{
${ }^{6}$ It is customary to associate the colour black with densities $\rho=1$ and the colour white with $\rho=0$. Intermediate values of $\rho(\boldsymbol{X})$ are described by means of a grey scale.
} 
where $K$ is the so-called convolution kernel and $\tilde{\rho}(\boldsymbol{X})$ is referred to as the filtered density field. An example of $K$ is that corresponding to the cone filter $K(r)=\max \{0,1-r / R\}$, with $R$ the filter radius. In addition, with the aim of reducing the appearance of intermediate densities, we use the classical smoothed Heaviside projection function proposed in [37]

$$
\hat{\rho}(\boldsymbol{X})=\frac{\tanh (\beta \eta)+\tanh (\beta(\tilde{\rho}(\boldsymbol{X})-\eta))}{\tanh (\beta \eta)+\tanh (\beta(1-\eta))},
$$

where $\hat{\rho}(\boldsymbol{X})$ is known as the physical density field and $\beta$ and $\eta$ are parameters carefully selected and updated throughout the optimisation process (for further details refer to [37]). Eventually, the interpolated strain energy density in (13) is replaced with

$$
e\left(\boldsymbol{X}, \boldsymbol{\nabla}_{0} \phi\right)=e^{\operatorname{SIMP}}\left(\hat{\rho}(\boldsymbol{X}), \nabla_{0} \phi\right):=(\hat{\rho}(\boldsymbol{X}))^{p} e^{\text {solid }}\left(\boldsymbol{\nabla}_{0} \phi\right)+\left[1-(\hat{\rho}(\boldsymbol{X}))^{p}\right] e^{\text {void }}\left(\boldsymbol{\nabla}_{0} \boldsymbol{\phi}\right),
$$

where the coefficient $p$ denotes a carefully selected integer number. In the case of linear elasticity, $p$ usually takes a value of 3 , as this ensures that the resulting interpolated elastic constants lie always within the Hashin-Strikman bounds [38]. To the best of authors' knowledge, analogous physical bounds for the case of nonlinear elasticity are not known and, hence, we will use $p=3$ in what follows. Finally, the volume constraint $g_{\Omega_{0}}(\chi)(15)$ is consistently replaced with $g_{\Omega_{0}}(\hat{\rho})$.

The first Piola-Kirchhoff stress tensor $\boldsymbol{P}$ associated with the strain energy density in (18) can be derived according to (8) as

$$
\boldsymbol{P}\left(\hat{\rho}(\boldsymbol{X}), \boldsymbol{\nabla}_{0} \boldsymbol{\phi}\right)=\partial_{\boldsymbol{\nabla}_{0} \phi} e^{\mathrm{SIMP}}\left(\hat{\rho}(\boldsymbol{X}), \boldsymbol{\nabla}_{0} \boldsymbol{\phi}\right)=(\hat{\rho}(\boldsymbol{X}))^{p} \boldsymbol{P}^{\text {solid }}\left(\boldsymbol{\nabla}_{0} \boldsymbol{\phi}\right)+\left[1-(\hat{\rho}(\boldsymbol{X}))^{p}\right] \boldsymbol{P}^{\mathrm{void}}\left(\boldsymbol{\nabla}_{0} \boldsymbol{\phi}\right),
$$

with

$$
\boldsymbol{P}^{\text {solid }}\left(\boldsymbol{\nabla}_{0} \boldsymbol{\phi}\right)=\partial_{\boldsymbol{\nabla}_{0} \phi} e^{\text {solid }}\left(\boldsymbol{\nabla}_{0} \boldsymbol{\phi}\right) ; \quad \boldsymbol{P}^{\mathrm{void}}\left(\boldsymbol{\nabla}_{0} \boldsymbol{\phi}\right)=\partial_{\boldsymbol{\nabla}_{0} \phi} e^{\text {void }}\left(\boldsymbol{\nabla}_{0} \boldsymbol{\phi}\right)=\varepsilon \mathcal{C}_{0}:\left(\boldsymbol{\nabla}_{0} \boldsymbol{\phi}-\boldsymbol{I}\right),
$$

and, analogously, the fourth order elasticity tensor $\mathcal{C}$ can be written (8) as

$$
\mathcal{C}\left(\hat{\rho}(\boldsymbol{X}), \nabla_{0} \boldsymbol{\phi}\right)=\partial_{\boldsymbol{\nabla}_{0} \phi \nabla_{0} \phi}^{2} e^{\operatorname{SIMP}}\left(\hat{\rho}(\boldsymbol{X}), \boldsymbol{\nabla}_{0} \boldsymbol{\phi}\right)=(\hat{\rho}(\boldsymbol{X}))^{p} \mathcal{C}^{\text {solid }}\left(\nabla_{0} \phi\right)+\left[1-(\hat{\rho}(\boldsymbol{X}))^{p}\right] \mathcal{C}^{\text {void }}\left(\boldsymbol{\nabla}_{0} \boldsymbol{\phi}\right),
$$

with

$$
\mathcal{C}^{\text {solid }}\left(\boldsymbol{\nabla}_{0} \phi\right)=\partial_{\boldsymbol{\nabla}_{0} \phi \nabla_{0} \phi}^{2} e^{\text {solid }}\left(\nabla_{0} \phi\right) ; \quad \mathcal{C}^{\text {void }}\left(\nabla_{0} \phi\right)=\partial_{\nabla_{0} \phi \nabla_{0} \phi}^{2} e^{\text {void }}\left(\nabla_{0} \phi\right)=\varepsilon \mathcal{C}_{0} .
$$

At last, we are now in a position to state the TO optimisation problem at hand, which can be recast as follows

$$
\left(P_{1}\right)\left\{\begin{array}{l}
\min _{\rho(\boldsymbol{X})} \mathcal{J}(\boldsymbol{\phi}), \\
\text { s.t }\left\{\begin{array}{l}
\text { State equations }(1),(4) ; \\
\text { Constitutive model }(18) ; \\
\text { Volume constraint } g_{\Omega_{0}}(\hat{\rho})(15) ; \\
0 \leq \rho(\boldsymbol{X}) \leq 1 .
\end{array}\right.
\end{array}\right.
$$

\subsection{The optimisation Lagrangian $\mathcal{L}$ : stationary conditions and descent direction}

Associated with the minimisation problem (23), the following Lagrangian functional $\mathcal{L}$ is defined

$$
\begin{aligned}
\mathcal{L}(\rho, \boldsymbol{\phi}, \boldsymbol{p}) & =\mathcal{J}(\boldsymbol{\phi})+\Pi(\rho, \boldsymbol{\phi}, \boldsymbol{p}) ; \\
\Pi(\rho, \boldsymbol{\phi}, \boldsymbol{p}) & =-\int_{\Omega_{0}} \boldsymbol{\nabla}_{0} \boldsymbol{p}: \boldsymbol{P}\left(\hat{\rho}(\rho(\boldsymbol{X})), \boldsymbol{\nabla}_{0} \boldsymbol{\phi}\right) d V+\int_{\Omega_{0}} \boldsymbol{f}_{0} \cdot \boldsymbol{p} d V+\int_{\partial_{N} \Omega_{0}} \boldsymbol{t}_{0} \cdot \boldsymbol{p} d A,
\end{aligned}
$$


where $\Pi(\rho, \boldsymbol{\phi}, \boldsymbol{p})$ represents the weak form of the equilibrium equations $(4), \boldsymbol{p}$ is the so-called adjoint state. Although not explicitly shown in the Lagrangian functional $(24)$, the volume constraint $g_{\Omega_{0}}(\hat{\rho})$ in (15) is enforced by means of the MMA method [39]. The directional derivative of the Lagrangian (excluding the volume contribution $g_{\Omega_{0}}(\hat{\rho})$ in $(15)$ ) with respect to an increment in the design density field $(\Delta \rho)$, namely the descent direction, yields

$$
D \mathcal{L}[\Delta \rho]=-\int_{\Omega_{0}}\left[\boldsymbol{\nabla}_{0} \boldsymbol{p}: \partial_{\hat{\rho}} \boldsymbol{P}\left(\hat{\rho}(\rho(\boldsymbol{X})), \nabla_{0} \boldsymbol{\phi}\right)\right] D \hat{\rho}[\Delta \rho] d V ; \quad D \hat{\rho}[\Delta \rho]=\frac{\partial \hat{\rho}}{\partial \tilde{\rho}} \frac{\partial \tilde{\rho}}{\partial \rho} \Delta \rho,
$$

where $\frac{\partial \hat{\rho}}{\partial \rho}$ and $\frac{\partial \tilde{\rho}}{\partial \rho}$ are obtained from (16)-(17), and $\partial_{\hat{\rho}} \boldsymbol{P}$ can be obtained by making use of (19) as

$$
\partial_{\hat{\rho}} \boldsymbol{P}\left(\hat{\rho}(\rho(\boldsymbol{X})), \boldsymbol{\nabla}_{0} \boldsymbol{\phi}\right)=p(\hat{\rho}(\boldsymbol{X}))^{p-1}\left(\boldsymbol{P}^{\text {solid }}\left(\boldsymbol{\nabla}_{0} \boldsymbol{\phi}\right)-\boldsymbol{P}^{\text {void }}\left(\boldsymbol{\nabla}_{0} \boldsymbol{\phi}\right)\right) .
$$

The mapping field $\boldsymbol{\phi}$ and the adjoint state field $\boldsymbol{p}$ can de deduced from the optimality conditions of the Lagrangian functional $\mathcal{L}$ in $(24)$, namely $D \mathcal{L}[\delta \boldsymbol{p}]=0$ and $D \mathcal{L}[\delta \boldsymbol{\phi}]=0$. The field $\boldsymbol{\phi}$ is obtained from the first optimality condition as

$$
D \mathcal{L}[\delta \boldsymbol{p}]=-\int_{\Omega_{0}} \boldsymbol{\nabla}_{0} \delta \boldsymbol{p}: \boldsymbol{P}\left(\hat{\rho}(\rho(\boldsymbol{X})), \nabla_{0} \boldsymbol{\phi}\right) d V+\int_{\Omega_{0}} \boldsymbol{f}_{0} \cdot \delta \boldsymbol{p} d V+\int_{\partial_{N} \Omega_{0}} \boldsymbol{t}_{0} \cdot \delta \boldsymbol{p} d A=0
$$

and the adjoint state $\boldsymbol{p}$ is obtained from the second optimality condition as

$$
D \mathcal{L}[\delta \phi]=\int_{\Omega_{0}} \boldsymbol{f}_{0} \cdot \delta \phi d V+\int_{\partial_{N} \Omega_{0}} \boldsymbol{t}_{0} \cdot \delta \phi d A-\int_{\Omega_{0}} \boldsymbol{\nabla}_{0} \boldsymbol{p}: \mathcal{C}\left(\hat{\rho}(\rho(\boldsymbol{X})), \boldsymbol{\nabla}_{0} \boldsymbol{\phi}\right): \boldsymbol{\nabla}_{0} \delta \boldsymbol{\phi} d V=0
$$

\subsection{Classical TO solution strategy}

The prototypical computational strategy used to find the solution (at each TO iteration) of the nonlinear equilibrium equations (i.e. weak form (27)) relies on the use of the Newton-Raphson method in conjunction with an incremental loading process. This procedure is typically employed until convergence problems arise (usually in initial and intermediate TO designs). At this juncture, an arc-length technique is introduced in order to attempt bypassing the onset of instability (buckling) phenomena and facilitate the convergence of the TO algorithm.

\section{A stabilisation methodology for SIMP-based TO}

It is well-known that the onset of instability phenomena in density-based methods (i.e. SIMP method) is due to (i) the development of regions with intermediate densities $[11,16,33]$ and (ii) the nature itself of the chosen energy interpolation (13), consisting of a convex combination of the polyconvex (non-convex) strain energy density of the solid phase $\left(e^{\text {solid }}\right)$ and the convex strain energy density of the void phase $\left(e^{\text {void }}\right)$. Indeed, in regions with very low densities, the strain energy density of the solid phase becomes negligible and it is the convex (stable) strain energy density of the void that is dominant. On the contrary, in intermediate density regions, the polyconvex solid component of the strain energy is not negligible, making these regions prone to exhibit geometric instabilities due to a combination of two reasons: (i) the loss of convexity of the strain energy functional of the solid phase and (ii) its weakened material parameters (which are pre-multiplied by low values of $\hat{\rho}(\rho(\boldsymbol{X}))$ ). In regions with high densities (with $\hat{\rho}(\rho(\boldsymbol{X}))$ approaching the upper bound 1), the latter reason (ii) stops being a problematic factor. 
Unfortunately, even sophisticated arc-length procedures (Section 3.2) cannot always resolve some of these instabilities (typically arising in initial and intermediate TO designs), which can ultimately result in the breakdown or non-convergence of the TO process. It is for this reason that a new approach is presented in this paper, whereby some of the concepts put forward in [1] in the context of level-set based TO are borrowed and carefully adapted for SIMP-based TO.

\subsection{Proposed incrementally linearised stabilisation methodology}

In the case of large deformations, it is customary to solve the nonlinear equilibrium equations in an incremental manner $n=0 \ldots N-1$, being $N$ the maximum number of load increments. The external forces $\left(\boldsymbol{f}_{0}\right.$ and $\boldsymbol{t}_{0}$ in $\left.(4)\right)$ are applied incrementally, their magnitude being controlled by an incremental load factor $\lambda_{n+1}$ at a given load increment $n+1$, such that $\lambda_{n+1}=(n+1) / N$. At load increment $n+1$, the incremental equilibrium equations can be formulated as

$$
\begin{aligned}
\operatorname{DIV} \boldsymbol{P}_{n+1}+\lambda_{n+1} \boldsymbol{f}_{0} & =\mathbf{0} ; & & \text { in } \Omega_{0} ; \\
\boldsymbol{P}_{n+1} \boldsymbol{N} & =\lambda_{n+1} \boldsymbol{t}_{0} ; & & \text { on } \partial_{N} \Omega_{0} ; \\
\boldsymbol{\phi} & =\overline{\boldsymbol{\phi}} ; & & \text { on } \partial_{\phi} \Omega_{0},
\end{aligned}
$$

where $\boldsymbol{P}_{n+1}$ denotes the first Piola-Kirchhoff stress tensor evaluated at load increment $n+1$, which can be consistently obtained from the strain energy density $\left.e^{\operatorname{SIMP}}\right|_{n+1}=e^{\operatorname{SIMP}}\left(\hat{\rho}\left(\rho(\boldsymbol{X}), \boldsymbol{\nabla}_{0} \boldsymbol{\phi}_{n+1}\right)\right.$ by using $(8)_{a}$.

With the objective of circumventing the numerical difficulties described in Section 3.2 with classical TO solution strategies, specially during initial and intermediate TO design stages, it seems reasonable to relax the exact satisfaction of the equilibrium equations [40]. With this in mind, following a similar approach to that presented by the authors in [1], the strain energy density $\left.e^{\text {SIMP }}\right|_{n+1}$ (used in the evaluation of $\boldsymbol{P}_{n+1}$ in (29)) is approximated by means of the following Taylor series expansion $\left.\left.e^{\mathrm{SIMP}}\right|_{n+1} \approx e_{\mathrm{incr}}^{\mathrm{SIMP}}\right|_{n+1}$ with

$$
\left.e_{\text {incr }}^{\mathrm{SIMP}}\right|_{n+1}:=\left.e^{\mathrm{SIMP}}\right|_{n}+\left.\boldsymbol{P}\right|_{n}: \nabla_{0} \Delta \boldsymbol{u}_{n+1}+\frac{1}{2} \nabla_{0} \Delta \boldsymbol{u}_{n+1}:\left.\mathcal{C}\right|_{n}: \nabla_{0} \Delta \boldsymbol{u}_{n+1} ; \quad \Delta \boldsymbol{u}_{n+1}=\boldsymbol{u}_{n+1}-\boldsymbol{u}_{n},
$$

where $\left.e^{\mathrm{SIMP}}\right|_{n}=e^{\mathrm{SIMP}}\left(\hat{\rho}(\rho(\boldsymbol{X})), \boldsymbol{\nabla}_{0} \boldsymbol{\phi}_{n}\right)$ is the interpolated energy at load increment $n$, and the first Piola-Kirchoff stress tensor $\left.\boldsymbol{P}\right|_{n}$ and the fourth order constitutive stress tensor $\left.\mathcal{C}\right|_{n}$ defined as

$$
\left.\boldsymbol{P}\right|_{n}:=\partial_{\nabla_{0} \phi} e^{\operatorname{SIMP}}\left(\hat{\rho}\left(\rho(\boldsymbol{X}), \boldsymbol{\nabla}_{0} \boldsymbol{\phi}_{n}\right)=\left.(\hat{\rho}(\boldsymbol{X}))^{p} \boldsymbol{P}^{\text {solid }}\right|_{n}+\left.\left[1-(\hat{\rho}(\boldsymbol{X}))^{p}\right] \boldsymbol{P}^{\text {void }}\right|_{n},\right.
$$

and

$$
\left.\mathcal{C}\right|_{n}:=\partial_{\boldsymbol{\nabla}_{0} \phi \boldsymbol{\nabla}_{0} \boldsymbol{\phi}}^{2} e^{\mathrm{SIMP}}\left(\hat{\rho}\left(\rho(\boldsymbol{X}), \boldsymbol{\nabla}_{0} \boldsymbol{\phi}_{n}\right)=\left.(\hat{\rho}(\boldsymbol{X}))^{p} \mathcal{C}^{\text {solid }}\right|_{n}+\left.\left[1-(\hat{\rho}(\boldsymbol{X}))^{p}\right] \mathcal{C}^{\text {void }}\right|_{n}\right.
$$

As previously shown by some of the authors in [1], the strain energy approximation $\left.e^{\mathrm{SIMP}}\right|_{n+1} \approx$ $\left.e_{\text {incr }}^{\text {SIMP }}\right|_{n+1}$ is perfectly valid provided that a reasonably small incremental $\Delta \boldsymbol{u}_{n+1}$ displacement is undergone within a load increment, which can be easily controlled by the number of user-defined load increments.

Another important ingredient put forward in [1], also adopted in this work, is the stabilisation of local instabilities characterised by the loss of positive definiteness of the elasticity tensor (which can result in the possible development of global instabilities). In order to accomplish this, we employ a regularisation 
(stabilisation) of the elasticity tensor at every load increment $n$ (i.e. $\left.\mathcal{C}^{\text {solid }}\right|_{n}$ in (31)). This is based on an additive perturbation of $\left.\mathcal{C}^{\text {solid }}\right|_{n}$ through a positive definite elasticity tensor $\left.\Delta \mathcal{C}_{\text {reg }}^{\text {solid }}\right|_{n}$ as follows

$$
\left.\mathcal{C}_{\text {reg }}^{\text {solid }}\right|_{n}:=\left.\mathcal{C}^{\text {solid }}\right|_{n}+\left.\Delta \mathcal{C}_{\text {reg }}^{\text {solid }}\right|_{n} ; \quad \boldsymbol{V}:\left.\mathcal{C}_{\text {reg }}^{\text {solid }}\right|_{n}: \boldsymbol{V} \geq 0 ; \quad \forall \boldsymbol{V} \in \mathbb{R}^{d \times d}, \boldsymbol{V} \neq \mathbf{0} .
$$

In (33), $\left.\Delta \mathcal{C}_{\text {reg }}^{\text {solid }}\right|_{n}$ must be defined such that positive definiteness of the regularised elasticity tensor at each load increment $n$, namely $\left.\mathcal{C}_{\text {reg }}^{\text {solid }}\right|_{n}$, is satisfied. We use the same technique as in [1], namely

$$
\left.\Delta \mathcal{C}_{\text {reg }}^{\text {solid }}\right|_{n}=(1+\delta) \Gamma \mathcal{I}
$$

where $\mathcal{I}$ is the fourth order identity tensor defined in $(11)_{a}$ and $\Gamma \in \mathbb{R}^{+}$such that all the leading minors of $\left(\left.\mathcal{C}^{\text {solid }}\right|_{n}+\Gamma \mathcal{I}\right)$ are non-zero. Therefore, the parameter $\Gamma$ helps stabilise the possible loss of convexity of the constitutive tensor. As in [1], an iterative bisection algorithm has been implemented in order to find the minimum value of $\Gamma$ complying with $(33)_{b}$. An additional parameter $\delta \in \mathbb{R}^{+}$is used in order to prevent the appearance of possible rigid body motions due to the presence of disconnected parts in the structure which can potentially arise during the TO process. For all the numerical simulations shown in this paper, a value $\delta=10^{-3}$ was used. It is important to emphasise that the regularisation of the constitutive tensor in $\left.\mathcal{C}_{\text {reg }}^{\text {solid }}\right|_{n}$ is a very localised effect which only takes place in very concentrated regions of the computational domain. Moreover, the amount of stabilisation added is the minimum possible to ensure the convexity of the global tangent operator (evaluated via the bisection algorithm). This will be later illustrated in the paper as part of numerical examples Section.

Finally, replacing $\left.\mathcal{C}^{\text {solid }}\right|_{n}$ with $\left.\mathcal{C}_{\text {reg }}^{\text {solid }}\right|_{n}$ in (31), the first Piola-Kirchhoff stress tensor $\boldsymbol{P}_{n+1}$ and the fourth order elasticity tensor can be obtained by computing the first and second derivatives of $\left.e_{\mathrm{incr}}^{\mathrm{SIMP}}\right|_{n+1}$ (30) according to (8) as

$$
\boldsymbol{P}_{n+1}=(\hat{\rho}(\boldsymbol{X}))^{p}\left(\left.\boldsymbol{P}^{\text {solid }}\right|_{n}+\left.\mathcal{C}_{\text {reg }}^{\text {solid }}\right|_{n}: \nabla_{0} \Delta \boldsymbol{u}_{n+1}\right)+\left[1-(\hat{\rho}(\boldsymbol{X}))^{p}\right]\left(\left.\boldsymbol{P}^{\text {void }}\right|_{n}+\mathcal{C}^{\text {void }}: \nabla_{0} \Delta \boldsymbol{u}_{n+1}\right),
$$

and

$$
\mathcal{C}_{n+1}=\left.(\hat{\rho}(\boldsymbol{X}))^{p} \mathcal{C}_{\text {reg }}^{\text {solid }}\right|_{n}+\left[1-(\hat{\rho}(\boldsymbol{X}))^{p}\right] \mathcal{C}^{\text {void }}
$$

Notice from (35) that the regularised first Piola-Kirchhoff stress tensor $\boldsymbol{P}_{n+1}$ is linear with respect to the unknown displacement field $\boldsymbol{u}_{n+1}$. This entails that the original nonlinear equilibrium equations are transformed into an approximate linear set for each load increment. Hence, the use of a Newton-Raphson method is not needed to solve (29).

Remark 2. An alternative manner to guarantee structural stability is the incorporation of buckling constraints within the optimisation problem [41]. However, the complexity and computational effort associated with this formulation is dramatically increased. Typically the buckling constraint is mathematically formulated as an eigenvalue problem on the (potentially very large) global stiffness matrix of the overall structure [41]. In addition, artificial instabilities induced by intermediate density regions need to be filtered out [41]. In this sense, the incrementally linearised regularisation TO formulation described above can be seen as a very efficient computationally alternative. 


\subsection{An incrementally linearised stabilisation TO solution strategy}

In this strategy, the numerical approach described above is applied for all load increments from $\lambda_{0}=0$ to $\lambda_{N}=1$ for every TO iteration ${ }^{7}$. Therefore, both pre- and post-buckling regions are approximated by means of the incrementally linearised stabilisation approach. As an interesting by-product, the use of a Newton-Raphson algorithm is completely circumvented, resulting in a very competitive algorithm from the computational speed standpoint. As such, the new minimisation problem is now defined as

$$
\left(P_{2}\right)\left\{\begin{array}{l}
\min _{\rho(\boldsymbol{X})} \mathcal{J}\left(\phi_{N}\right), \\
\text { s.t }\left\{\begin{array}{l}
\text { State equations }(1),(29) \text { at every load increment } n=0 \ldots N-1 ; \\
\text { Regularised constitutive model: (18), (30) and }(33) ; \\
\text { Volume constraint } g_{\Omega_{0}}(\hat{\rho})(15) ; \\
0 \leq \rho(\boldsymbol{X}) \leq 1 .
\end{array}\right.
\end{array}\right.
$$

Associated with the minimisation problem (37), the following regularised Lagrangian functional $\mathcal{L}^{\text {reg }}$ can be introduced ${ }^{8}$

$$
\mathcal{L}^{\mathrm{reg}}\left(\rho,\left\{\boldsymbol{\phi}_{1}, \ldots, \boldsymbol{\phi}_{N+1}\right\},\left\{\boldsymbol{p}_{1}, \ldots, \boldsymbol{p}_{N+1}\right\}\right)=\sum_{n=0}^{N-1} \mathcal{L}_{n+1}^{\mathrm{reg}}\left(\rho, \boldsymbol{\phi}_{n+1}, \boldsymbol{p}_{n+1}\right),
$$

where each of the regularised Lagrangian functionals $\mathcal{L}_{n+1}^{\text {reg }}$ at every load increment $n+1$ is defined as

$$
\mathcal{L}_{n+1}^{\mathrm{reg}}\left(\rho, \boldsymbol{\phi}_{n+1}, \boldsymbol{p}_{n+1}\right)=\left\{\begin{array}{ll}
\Pi\left(\rho, \boldsymbol{\phi}_{n+1}, \boldsymbol{p}_{n+1}\right) & \text { if } n+1<N \\
\mathcal{J}\left(\boldsymbol{\phi}_{n+1}\right)+\Pi\left(\rho, \boldsymbol{\phi}_{n+1}, \boldsymbol{p}_{n+1}\right) & \text { if } n+1=N
\end{array},\right.
$$

with

$$
\Pi\left(\rho, \boldsymbol{\phi}_{n+1}, \boldsymbol{p}_{n+1}\right)=-\int_{\Omega_{0}} \boldsymbol{\nabla}_{0} \boldsymbol{p}_{n+1}: \boldsymbol{P}_{n+1} d V+\lambda_{n+1}\left(\int_{\Omega_{0}} \boldsymbol{f}_{0} \cdot \boldsymbol{p} d V+\int_{\partial_{N} \Omega_{0}} \boldsymbol{t}_{0} \cdot \boldsymbol{p} d A\right),
$$

where $\Pi\left(\rho, \boldsymbol{\phi}_{n+1}, \boldsymbol{p}_{n+1}\right)$ represents the weak form of the equilibrium system (29) at every load increment $n+1$ and $\boldsymbol{p}_{n+1}$, the adjoint state at load increment $n+1$. The directional derivative of the regularised Lagrangian with respect to an increment in the design density variable $\Delta \rho$, namely the descent direction, yields

$$
D \mathcal{L}^{\mathrm{reg}}[\Delta \rho]=\sum_{n=0}^{N-1} D \mathcal{L}_{n+1}^{\mathrm{reg}}[\Delta \rho]=-\sum_{n=0}^{N-1} \int_{\Omega_{0}} \boldsymbol{\nabla}_{0} \boldsymbol{p}_{n+1}: \partial_{\hat{\rho}} \boldsymbol{P}_{n+1} D \hat{\rho}[\Delta \rho] .
$$

Furthermore, $\partial_{\hat{\rho}} \boldsymbol{P}_{n+1}$ can be obtained making use of (35) as

$$
\partial_{\hat{\rho}} \boldsymbol{P}_{n+1}=p(\hat{\rho}(\boldsymbol{X}))^{p-1}\left(\left.\boldsymbol{P}^{\text {solid }}\right|_{n}-\left.\boldsymbol{P}^{\text {void }}\right|_{n}+\left(\left.\mathcal{C}_{\text {reg }}^{\text {solid }}\right|_{n}-\mathcal{C}^{\text {void }}\right): \nabla_{0} \Delta \boldsymbol{u}_{n+1}\right) .
$$

\footnotetext{
${ }^{7}$ Importantly, the performance of the final design at $\lambda_{N}=1$ is always tested at a post-processing stage with the classical TO strategy in Section 3.2, ensuring thus the exact satisfaction of the equilibrium equations for the final TO stage.

${ }^{8}$ Although not explicitly shown in the Lagrangian functional (38), the volume constraint $g_{\Omega_{0}}(\hat{\rho})$ in (15) is enforced by means of the MMA method [39].
} 
The mapping field $\boldsymbol{\phi}_{n+1}$ and the adjoint state field $\boldsymbol{p}_{n+1}$ can de deduced from the optimality conditions of the Lagrangian functional $\mathcal{L}^{\text {reg }}$ in (39), namely $D \mathcal{L}^{\text {reg }}\left[\delta \boldsymbol{p}_{n+1}\right]=0$ and $D \mathcal{L}^{\text {reg }}\left[\delta \boldsymbol{\phi}_{n+1}\right]=0$. The field $\phi_{n+1}$ is obtained from the first optimality condition as

$$
\begin{aligned}
D \mathcal{L}^{\mathrm{reg}}\left[\delta \boldsymbol{p}_{n+1}\right]=D \mathcal{L}_{n+1}^{\mathrm{reg}}\left[\delta \boldsymbol{p}_{n+1}\right] & =-\int_{\Omega_{0}} \boldsymbol{\nabla}_{0} \delta \boldsymbol{p}_{n+1}: \boldsymbol{P}_{n+1} d V \\
& +\lambda_{n+1}\left(\int_{\Omega_{0}} \boldsymbol{f}_{0} \cdot \delta \boldsymbol{p}_{n+1} d V+\int_{\partial_{N} \Omega_{0}} \boldsymbol{t}_{0} \cdot \delta \boldsymbol{p}_{n+1} d A\right)=0,
\end{aligned}
$$

and the adjoint state $\boldsymbol{p}_{n+1}$ is obtained from the second optimality condition as

$$
D \mathcal{L}^{\mathrm{reg}}\left[\delta \boldsymbol{\phi}_{n+1}\right]=D \mathcal{L}_{n+1}^{\mathrm{reg}}\left[\delta \boldsymbol{\phi}_{n+1}\right]= \begin{cases}-\int_{\Omega_{0}} \boldsymbol{\nabla}_{0} \boldsymbol{p}_{n+1}: \mathcal{C}_{n+1}: \boldsymbol{\nabla}_{0} \delta \boldsymbol{\phi}_{n+1} d V=0 . & \text { if } n+1<N \\ \int_{\Omega_{0}}^{\boldsymbol{f}_{0} \cdot \delta \boldsymbol{\phi}_{n+1} d V+\int_{\partial_{N} \Omega_{0}} \boldsymbol{t}_{0} \cdot \delta \boldsymbol{\phi}_{n+1} d A} \\ -\int_{\Omega_{0}} \boldsymbol{\nabla}_{0} \boldsymbol{p}_{n+1}: \mathcal{C}_{n+1}: \boldsymbol{\nabla}_{0} \delta \boldsymbol{\phi}_{n+1} d V=0 \quad \text { if } n+1=N\end{cases}
$$

From (44), it is possible to observe that the adjoint state $\boldsymbol{p}_{n+1}$ vanishes for all load increments except for the last, i.e. $\boldsymbol{p}_{n+1}=\mathbf{0}$ (if $n+1<N$ ). Hence, the descent direction in (45) reduces to

$$
D \mathcal{L}^{\mathrm{reg}}[\Delta \rho]=D \mathcal{L}_{N}^{\mathrm{reg}}[\Delta \rho]=-\int_{\Omega_{0}} \boldsymbol{\nabla}_{0} \boldsymbol{p}_{N}: \partial_{\hat{\rho}} \boldsymbol{P}_{N} D \hat{\rho}[\Delta \rho] .
$$

\subsection{A hybrid TO solution strategy}

In this strategy, within a given TO iteration, the classical TO solution strategy (refer to Section 3.2) is used until a critical point (before the ultimate design load is attained) arises (i.e. $\lambda_{n+1}=\lambda_{c r}<1$ ). From this instant onwards $\lambda_{n+1} \in\left(\lambda_{c r}, 1\right]$, a switch is made to the incrementally linearised regularisation TO solution strategy (refer to Section 4.1).

This hybrid TO solution strategy is particularly suitable towards the end of the TO process, when the current structural design is mostly devoid from intermediate density regions or, equivalently, when the updated contrast parameter $\beta$ in (17) reaches a sufficiently large value, yielding a sharper density projection. At this TO design stage, when the physical density approaches its lower and upper bounds ( 0 and 1 , respectively), it seems physically reasonable to demand a higher accuracy in the satisfaction of the equilibrium equations (at least in the pre-instability or pre-buckling region) and classical TO solution strategies should be used. Naturally, for final designs completely devoid of structural buckling, the hybrid TO solution strategy will nor require making use of the switch and the classical TO solution strategy can be used for the entire loading process. 


\section{Numerical examples}

The objective of this Section is to demonstrate the performance and applicability of the two new TO methodologies presented above for a series of problems of interest. Specifically: (i) the incrementally linearised stabilisation TO solution strategy in Section 4.2 (Reg-TO strategy) and (ii) the hybrid TO solution strategy in Section 4.3 (Hybrid-TO strategy). Notice that the classical TO solution strategy shown in Section 3.2 was not found robust enough to conclude the TO process for the challenging examples analysed and, hence, its results are not included. Three classical two-dimensional plane stress benchmark examples will be thoroughly studied before presenting a large scale three-dimensional example. For all the examples presented, the standard approach (arc-length or Newton-Raphson) did not converge. The Hybrid-TO approach only adds stabilisation at intermediate TO iterations, where the standard approach does not converge. The Reg-TO approach is merely presented as a very efficient (computationally time-wise) alternative that can potentially be used in TO. The performance of all the final designs obtained by means of both Hybrid-TO and Reg-TO has been tested at a postprocessing stage without adding any source of artificial stiffness. Specifically, an arc-length Newton-Raphson method has been employed in conjunction with a ratio of the material parameters for void and solid regions of $10^{-12}$. The performance of some of the designs is also shown well-beyond the design load just for display purposes.

\subsection{Numerical example 1}

The objectives of this example are

- O1 To test the performance of both Reg-TO and Hybrid-TO strategies for the so-called MBB problem [14], a classical problem in the field of Topology Optimisation, but presented in this case for a scenario with extremely large deformations.

- O2 To study the effect of the numerical stabilisation in the overall behaviour of the final structural design.

- O3 To study the effect on the accuracy of the number of load increments in the pre- and postbuckling regimes.

The geometry and boundary conditions for the problem are displayed in Figure 2 and the geometrical parameters, material properties, material parameters and simulation parameters are included in Table 1. Figure 3 shows the designs obtained with the Reg-TO and Hybrid-TO approaches for various design loads. The number of load increments used for the Reg-TO approach is $N=30$ (for all loading scenarios). In addition, the Hybrid-TO approach has been employed for all TO iterations (with the number of load increments beyond the onset of the critical point computed based on a pro-rata basis with respect to $N=30$ ).

The use of the Hybrid-TO approach for all TO iterations is not necessarily the most efficient strategy. As it will be shown later in the examples, it is preferable to start with the Reg-TO approach for the initial and intermediate TO iterations and only use the Hybrid-TO strategy towards the end of the TO design process, as this saves a considerable amount of computational time without jeopardising the quality of the final design. In any case, and for completeness, we advocate only in this example for the use of the Hybrid-TO strategy throughout the entire TO process.

The designs obtained by means of both strategies are qualitatively very similar and this also translates into a similar performance in terms of overall compliance. Figure 3 also displays the equilibrium paths 


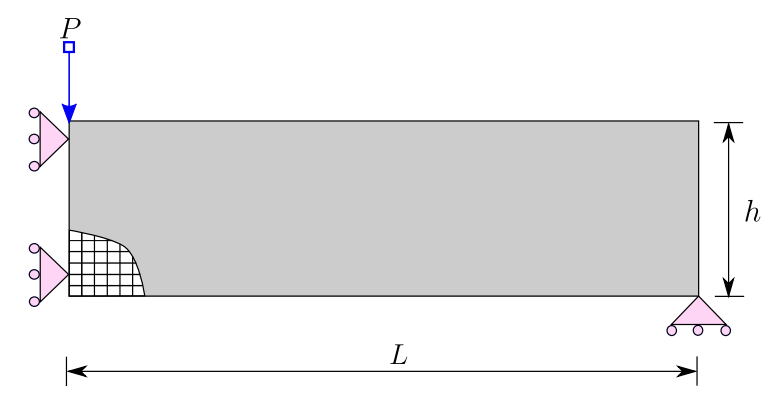

Figure 2: Numerical example 1. Boundary conditions.

Table 1: Numerical example 1. Material properties (see (7)), material parameters in (6) and simulation parameters.

\begin{tabular}{|c|c|c|c|c|c|c|}
\hline Geometrical parameters & $\begin{array}{c}L \\
h \\
t\end{array}$ & $\begin{array}{c}1 \\
0.33 \\
0.1\end{array}$ & $\begin{array}{l}\mathrm{m} \\
\mathrm{m} \\
\mathrm{m}\end{array}$ & Material Properties & $\begin{array}{c}\left.E\right|_{0} \\
\left.\nu\right|_{0}\end{array}$ & $\begin{array}{c}1 \\
0.4\end{array}$ \\
\hline Material Parameters & $\begin{array}{c}\mu_{1} \\
\mu_{2} \\
\lambda\end{array}$ & $\begin{array}{c}0.357 \\
0 \\
1.429\end{array}$ & $\begin{array}{l}\mathrm{Pa} \\
\mathrm{Pa} \\
\mathrm{Pa}\end{array}$ & Simulation Parameters & $\begin{array}{l}N_{x} \\
N_{y} \\
V^{*}\end{array}$ & $\begin{array}{c}180 \\
60 \\
0.4 \cdot V_{0}\end{array}$ \\
\hline
\end{tabular}

of the final designs: green line for Hybrid-TO and red line for Reg-TO. It is worth emphasising that the equilibrium paths have been obtained at a post-processing level, using an arc-length and, hence, ensuring that no artificial stiffness is introduced. The vertical axis displays the absolute value of the applied force $P$ (with the design load represented with a blue horizontal line) and the horizontal axis shows the absolute value of the displacement along the direction of the applied force and at its point of application. As it can be observed, in most figures (except Figure $3_{b}$ ), the value of the displacement attained for the prescribed design load value is extremely similar.

Figure $3_{b}$ (refer to equilibrium path) is the only one where Reg-TO yields a larger value of the displacement for the prescribed design load. In this case, the design experiences a softening near the design load (at around 90\% of its value) and the remaining 3 load increments are used to approximate the softening part of the equilibrium path via the Taylor series expansion (30). This small number of load increments is not enough taking into account the important variations in stiffness that occur during this softening region and thus a much greater number of load increments would be advisable. As a result, it is in these sort of scenarios, that we propose the use of a continuation strategy, whereby following the design obtained with the Reg-TO strategy, this is used as initial seed to launch a (quickly convergent) second TO phase resorting to the Hybrid-TO strategy, with initial value for the parameter $\beta$ in (17) the outcome of the previous Reg-TO phase.

In Figures $4_{a, b, c}$, the final designs corresponding to the first and second stages of this continuation TO process are shown overlapped. Clearly, the second TO stage leads to an extremely similar design to that of the first TO stage. Indeed, the only design difference lies in the increased thickness of the upper structural joints. However, this slight modification is sufficient to give a final design stable for the design load (see Figures $4_{d, e}$ ). Finally, Figure $4_{f}$ shows the equilibrium paths for the design obtained after the first TO stage (red line) and for that obtained after the second TO stage (blue line). The equilibrium paths of both designs are practically identical up to the first squared sign (close to the design load). 


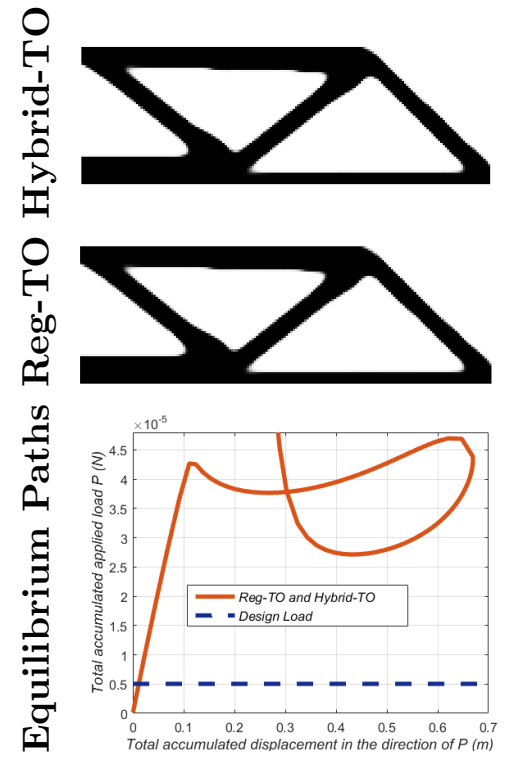

(a)

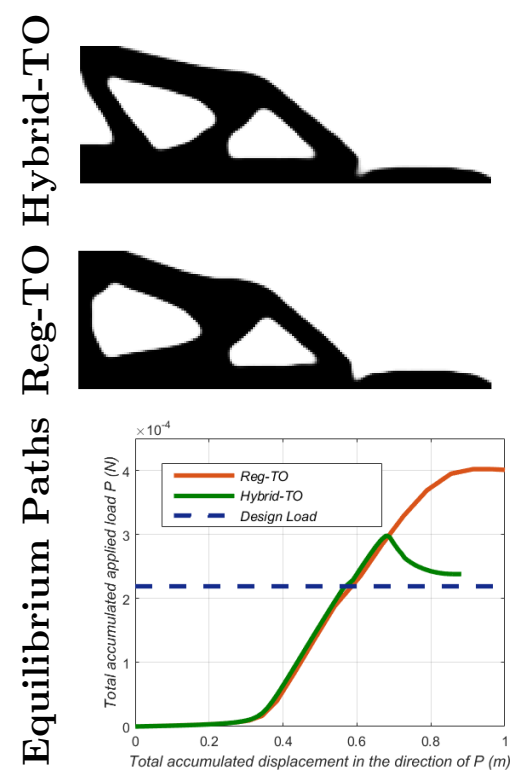

(e)
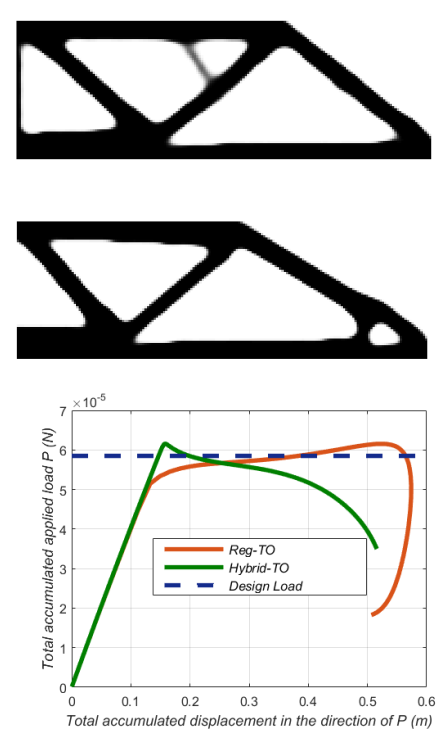

(b)
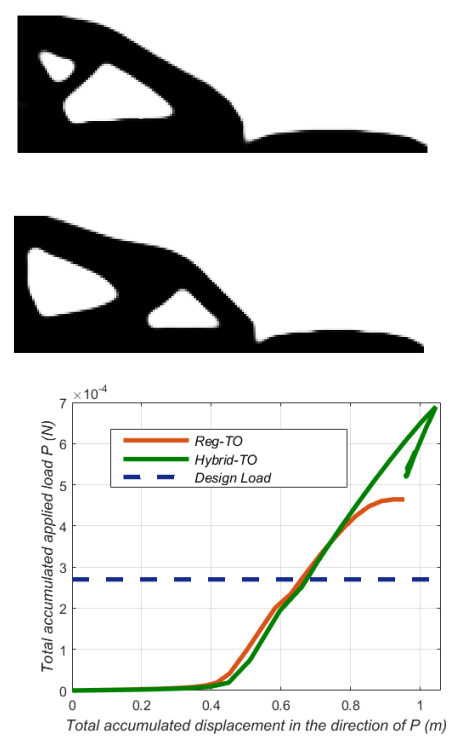

(f)
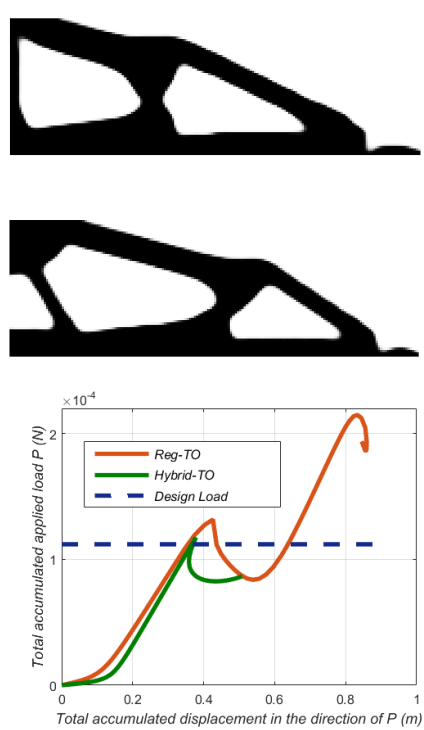

(c)
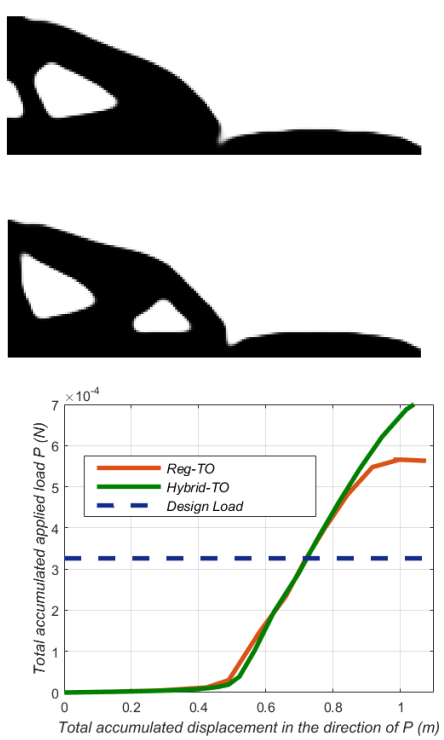

(g)
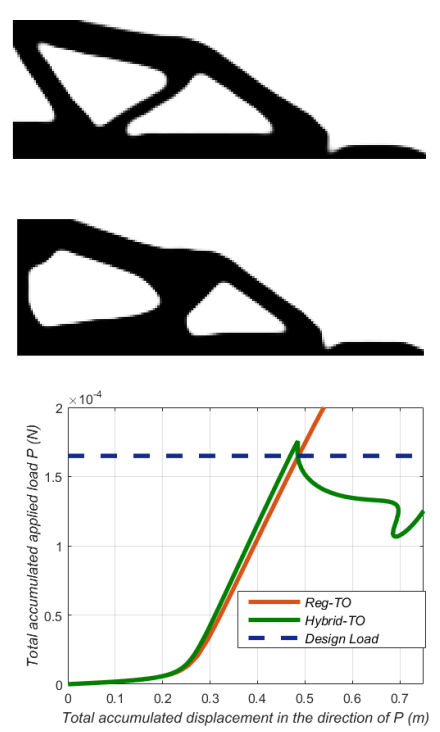

(d)
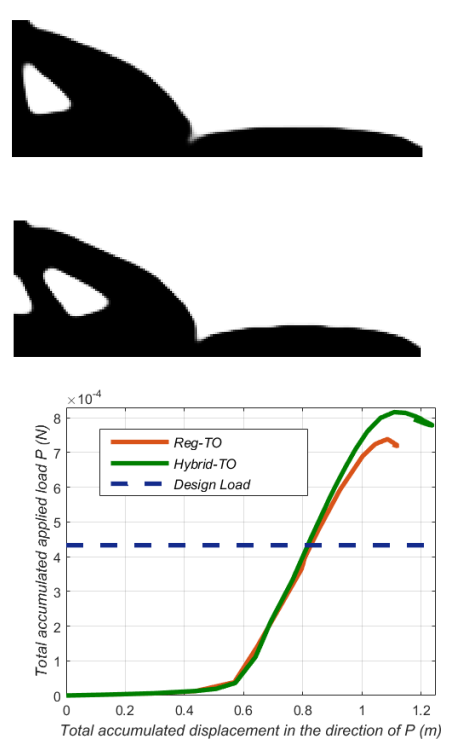

(h)

Figure 3: Numerical example 1. Optimal designs obtained by means of Hybrid-TO and Reg-TO strategies for loads: $P=\{0.5,5.85,11.2,16,22,27,32,43\} \times 10^{-5}$. Equilibrium paths for both strategies obtained by means of an arc-length without adding artificial stabilisation. 
From this point onwards, both designs behave very differently. The red line shows a softening response of the structure beyond this point, followed by an intricate equilibrium path after the third squared sign. On the other hand, the blue line shows a practically constant stiffening of the structure up to the design load, which is almost coincident with the critical point.

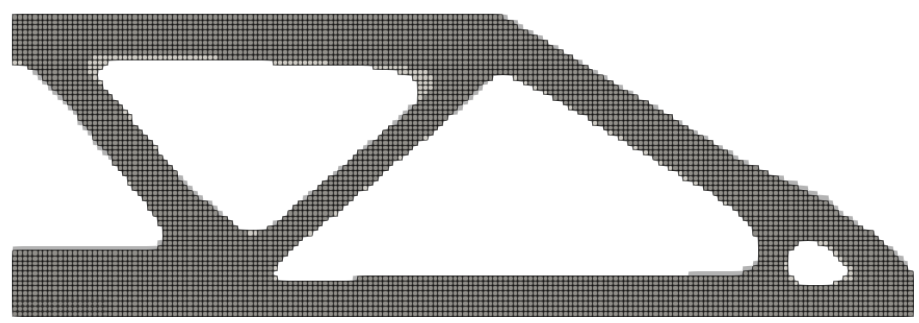

(a)

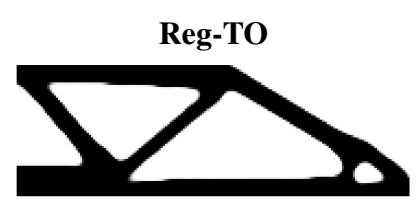

(b)

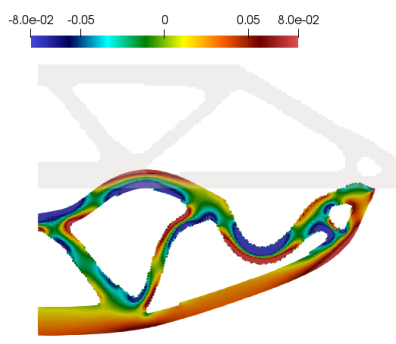

(d)

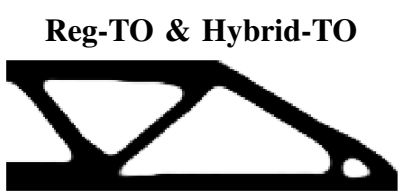

(c)

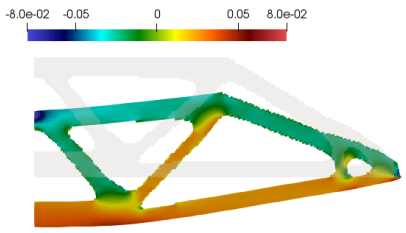

(e)

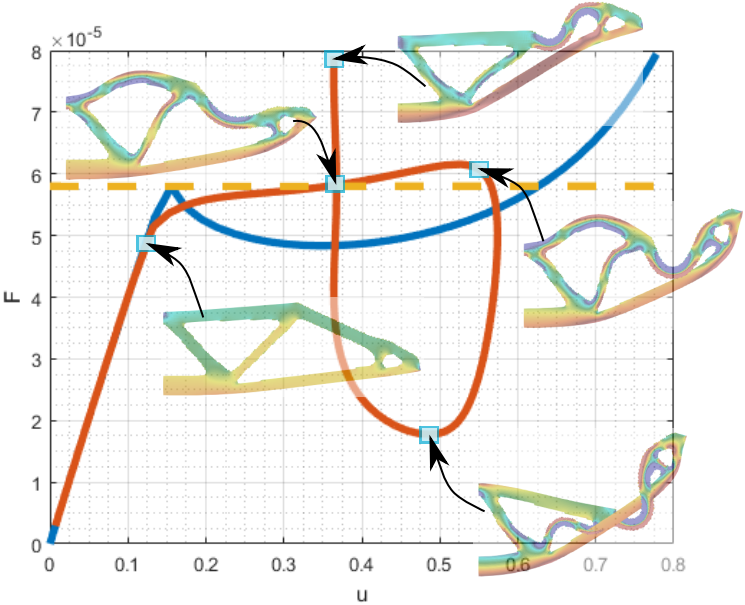

(f)

Figure 4: Numerical example 1. (a) Overlapped optimal designs obtained by means of Reg-TO (black-shadowed) and when continuated with Hybrid-TO (grey shadowed). (b)-(c) optimal designs obtained by means of Reg-TO and when continuated with Hybrid-TO; (d)-(e) Deformed configuration and pressure contour plot distribution for designs obtained with both approaches at the design load. (f) Equilibrium paths for designs in Figure $3 b$. Red line corresponds to design obtained by means of Reg-TO. Blue line corresponds to design obtained by means of Reg-TO and continuated with Hybrid-TO. Equilibrium paths obtained by means of arc-length without adding any source of artificial stiffness. The yellow horizontal dashed-line corresponds with the design load.

Figure 5 shows the deformed configurations and contour plot distributions of the hydrostatic pressure $p^{9}$ for the optimum designs obtained with Reg-TO and Hybrid-TO strategies for design loads $P=$ $\{0.5,5.85,11.2,16,22,27,32,43\} \times 10^{-5}$, where a reasonable agreement is observed between both $\mathrm{TO}$ strategies.

Finally, in order to address objective O2, Figure 6 illustrates the contour plot distribution of the dimensionless stabilisation indicator $s$, defined as

$$
s=\left\|\left.\Delta \mathcal{C}_{\mathrm{reg}}\right|_{n}\right\| /\left\|\left.\mathcal{C}\right|_{0}\right\|
$$

\footnotetext{
${ }^{9}$ The hydrostatic pressure, computed as $p=\frac{1}{3} \operatorname{tr}\left(J \boldsymbol{P} \boldsymbol{F}^{-T}\right)$, is a very common stress measure used in non-linear solid mechanics $[1,13]$.
} 

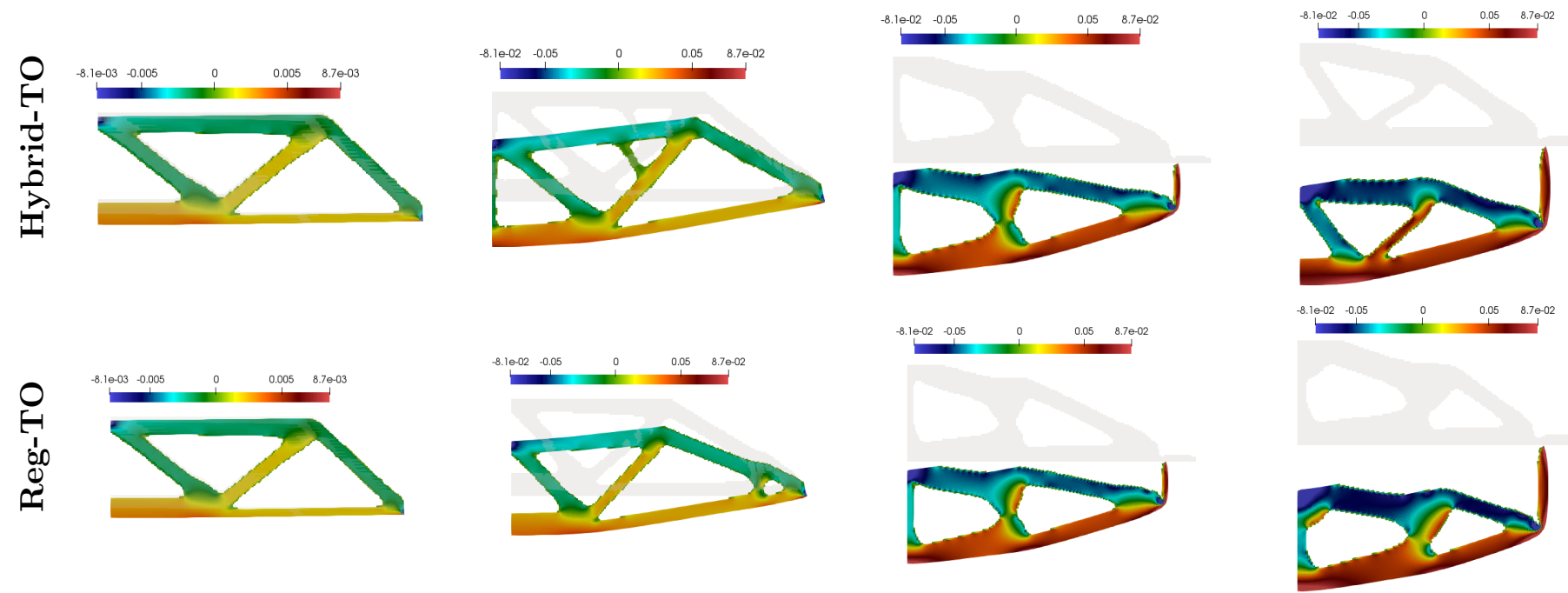

(a)

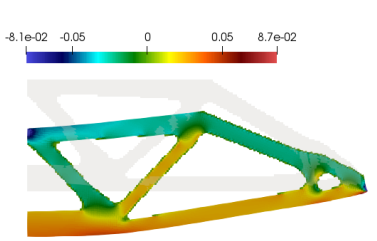

(c)

(d)
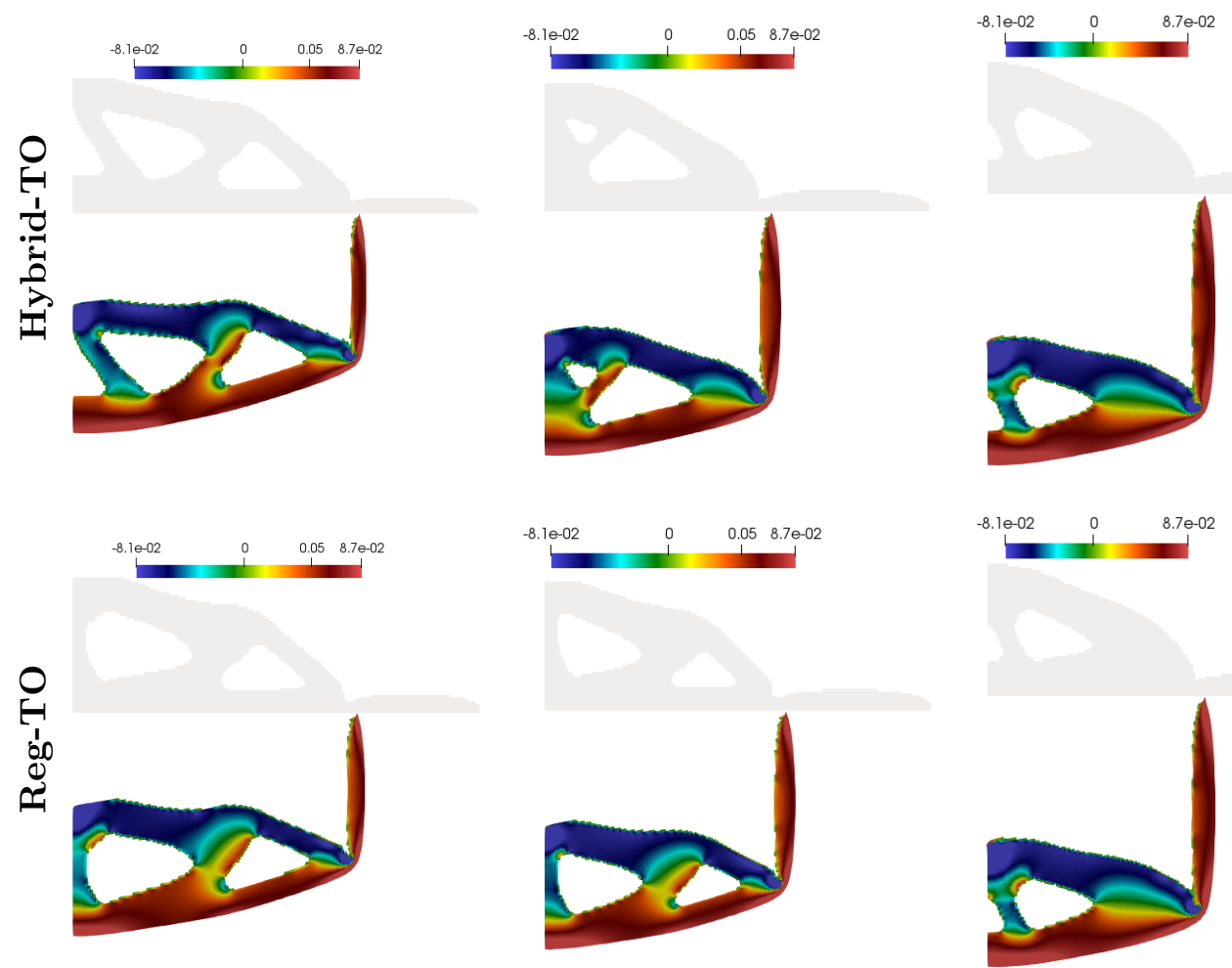

(e)

(f)

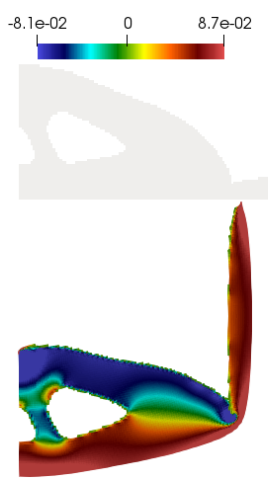

(g)
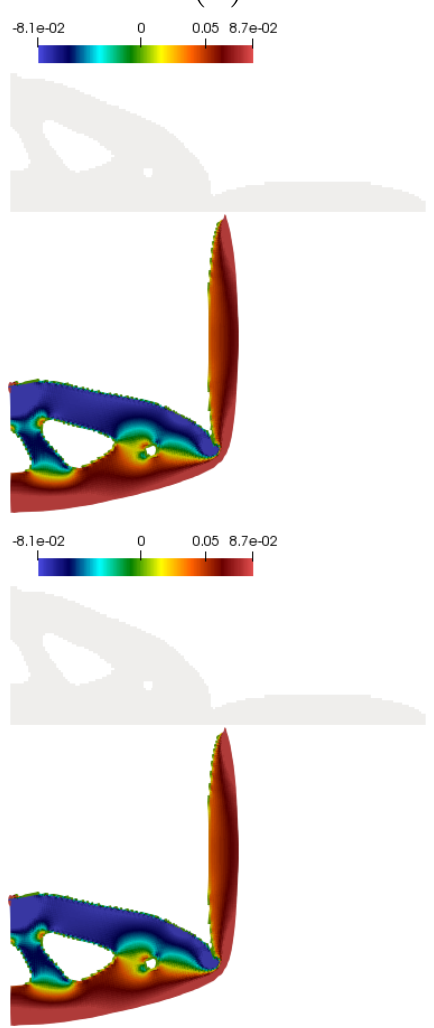

(h)

Figure 5: Numerical example 1. Contour plot distribution of the hydrostatic pressure $p$ associated with the designs obtained in Figure 3 for values of $P=\{0.5,5.85,11.2,16,22,27,32,43\} \times 10^{-5}$ (from left to right), using Hybrid-TO and Reg-TO strategies. 
where $\left.\Delta \mathcal{C}_{\text {reg }}\right|_{n}$ represents the stabilising part of the regularised elasticity tensor (34) and $\left.\mathcal{C}\right|_{0}$ denotes the elasticity tensor in the origin (i.e. $\boldsymbol{F}=\boldsymbol{I}$ ). Hence, the indicator $s$ is a relative measure of the amount of stabilisation required with respect to the elasticity tensor in the origin. The first configuration displayed in Figure 6 corresponds to $83.3 \%$ of the design load, and it coincides with the first squared sign on the red line equilibrium path in Figure $4_{f}$. For this load, a peak value for the stabilisation indicator of $s=0.14$ is obtained at the upper left corner of the structure, being practically zero elsewhere. This perfectly showcases that the stabilisation acts locally only, where positive definiteness of the elasticity tensor is compromised. Hence, stabilisation is restricted to the regions where it is strictly necessary. The three remaining configurations shown in the Figure correspond to larger values of the applied design load. In these cases, local instabilities start developing. Clearly, the stabilisation indicator only is activated in these (unstable) areas, reaching higher peak values of $s$ and remaining practically zero elsewhere.

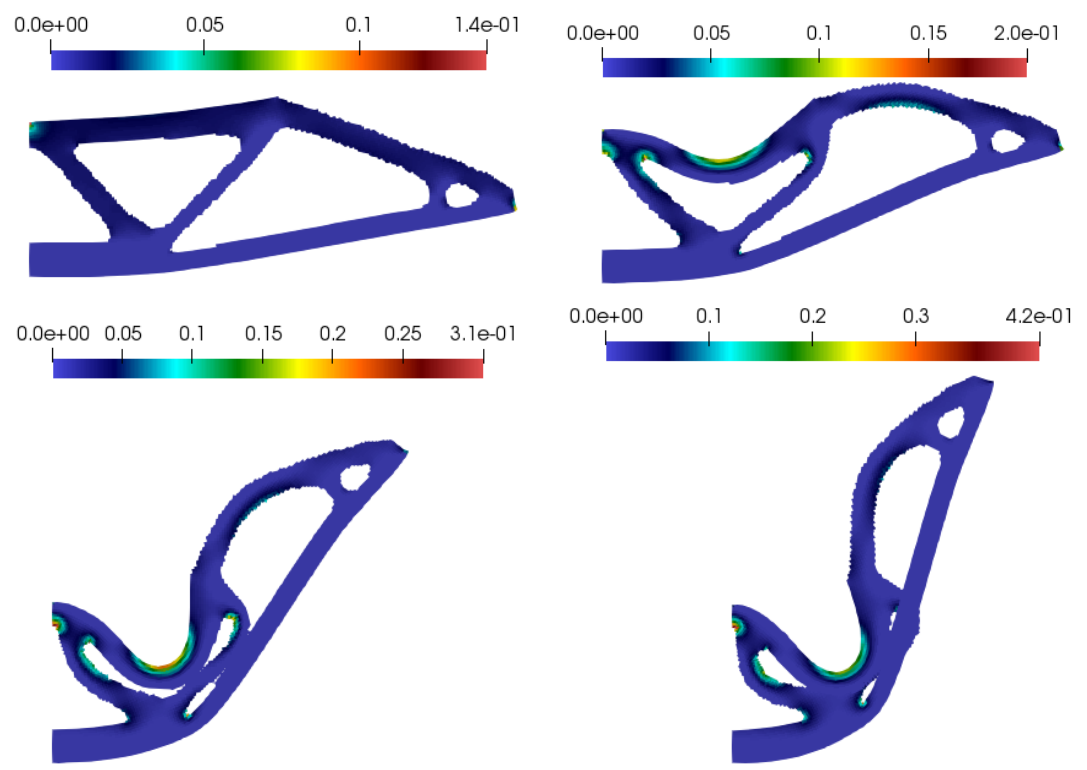

Figure 6: Numerical example 1. Relative stabilisation indicator $s$ in (46) for different values of the external applied load for design obtained with Reg-TO strategy.

In addition, Figure 7 shows the spherical parametrisation of the non-stabilised elasticity tensor $\left.\mathcal{C}\right|_{n}$ in a region where the stabilisation indicator $s$ reaches its maximum value. Furthermore, its regularised counterpart $\left.\mathcal{C}_{\text {reg }}^{\text {solid }}\right|_{n}$ and the purely stabilisation component of the latter $\left.\Delta \mathcal{C}_{\text {reg }}^{\text {solid }}\right|_{n}$ (see equations (33) and (34)) are also displayed. For a spherically parametrised vector $\boldsymbol{U}$, spherically parametrised elasticity tensor $\mathcal{C}(\mathcal{C})$ is defined as

$$
\mathcal{C}(\mathcal{C})=\boldsymbol{A}: \mathcal{C}: \boldsymbol{A} ; \quad \boldsymbol{A}=\boldsymbol{F U} \otimes \boldsymbol{U} ; \quad \boldsymbol{U}=\left[\begin{array}{lll}
\sin \beta \cos \alpha & \sin \beta \sin \alpha & \cos \beta
\end{array}\right]^{T},
$$

with $0 \leq \beta \leq 2 \pi$ and $0 \leq \alpha \leq \pi$. Notice that as this example is (plane stress) two-dimensional, for the sake of visualisation, we have re-constructed the three-dimensional elasticity tensor. This can be done by noting that the three-dimensional deformation gradient tensor $\boldsymbol{F}$ can be defined in terms of the in-plane deformation gradient tensor $\boldsymbol{F}_{2 D}$ and the out of plane thickness stretch $\lambda_{3}$ as

$$
\boldsymbol{F}=\left[\begin{array}{cc}
\boldsymbol{F}_{2 D} & \mathbf{0}_{2 \times 1} \\
\mathbf{0}_{1 \times 2} & \lambda_{3}
\end{array}\right]
$$


where $\lambda_{3}$ is the solution of the plane stress condition (the reader is referred to [1] for a detailed review of plane stress in the context of polyconvex elasticity). It is worth noticing that the isotropic nature of the stabilisation proposed in equation (34) yields a spherical shape for the shear modulus associated with the stabilising part $\left.\Delta \mathcal{C}_{\text {reg }}^{\text {solid }}\right|_{n}$, namely $\mathcal{C}\left(\left.\Delta \mathcal{C}_{\text {reg }}^{\text {solid }}\right|_{n}\right)$.

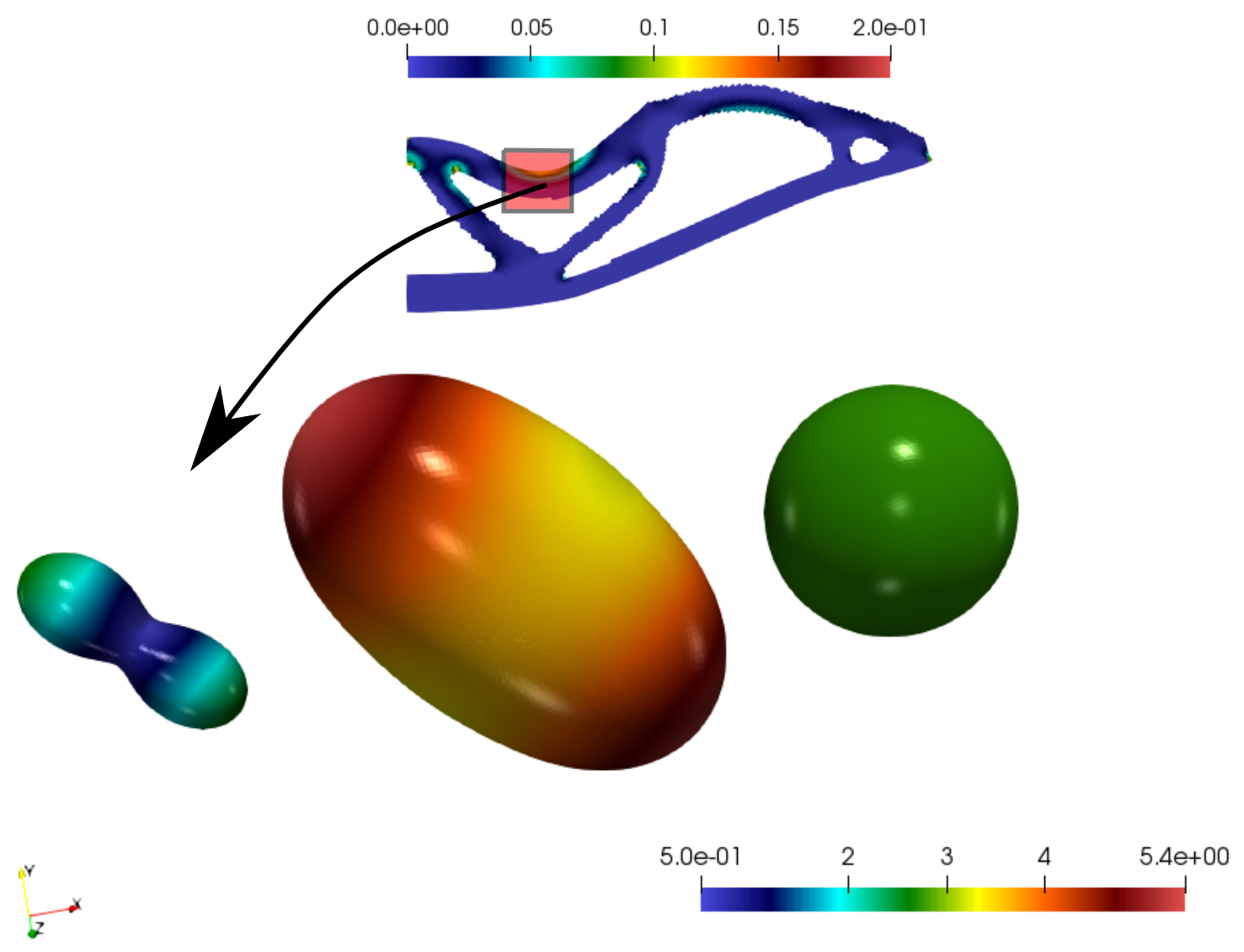

Figure 7: Numerical example 1. Spherical parametrisation of: (left) elasticity tensor $\left.\mathcal{C}^{\text {solid }}\right|_{n}$, namely $\mathcal{C}\left(\left.\mathcal{C}^{\text {solid }}\right|_{n}\right) ;($ center $)$ regularised elasticity tensor $\left.\mathcal{C}_{\text {reg }}^{\text {solid }}\right|_{n}$, namely $\mathcal{C}\left(\left.\mathcal{C}_{\text {reg }}^{\text {solid }}\right|_{n}\right) ;($ right $)$ stabilising contribution $\left.\Delta \mathcal{C}_{\text {reg }}^{\text {solid }}\right|_{n}$, namely $\mathcal{C}\left(\left.\Delta \mathcal{C}_{\text {reg }}^{\text {solid }}\right|_{n}\right)$.

In order to address objective $\mathbf{O 3}$, we first consider the design obtained in Figure $3_{f}$, which exhibits a very nonlinear equilibrium path in the pre-buckling regime, comprising an initial zone with low stiffness followed by a stiffening prior to the limit point. We will analyse this design using the incrementally linearised stabilised (Reg-TO) approach described throughout Section 4.2 for different values of the number of load increments. The applied load corresponds to the design load. In order to evaluate the accuracy of this methodology in the pre-buckling regime, the relative error of the vertical displacement $u_{\text {lin }}$ obtained for the various number of load increments in the point of application of the nodal load is computed as

$$
\text { Error }=\frac{u_{l i n}-u_{a l}}{u_{a l}} \times 100
$$

where $u_{a l}$ represents the displacement in the same point obtained by means of the arc-length method (true physical displacement). Clearly, despite the clear non-linearity of the equilibrium path, 30 load increments yield a relative error of $4 \%$, which is acceptable from the engineering standpoint. As expected, 
the error decreases as the number of load increments increases (see Figure $8_{a}$ ).

It is also important to observe the joint effect of the number of load increments and the stabilisation in the post-buckling regime. For that, we consider the design in Figure $3_{b}$ and a value of the applied load considerably higher than the design load and that the limit point. From Figure $8_{b}$, as the number of load increments increases, the stabilised equilibrium path tends to a horizontal line (a negative slope is prevented by the stabilisation). Interestingly, once the true equilibrium path (obtained by means of the arc-length method, see black curve on the same figure) reaches a value above the limit point for the second time, the stabilised equilibrium paths tend to coincide with the true physical equilibrium path as the number of load increments is increased (see zoomed detail in Figure $8_{b}$ ).
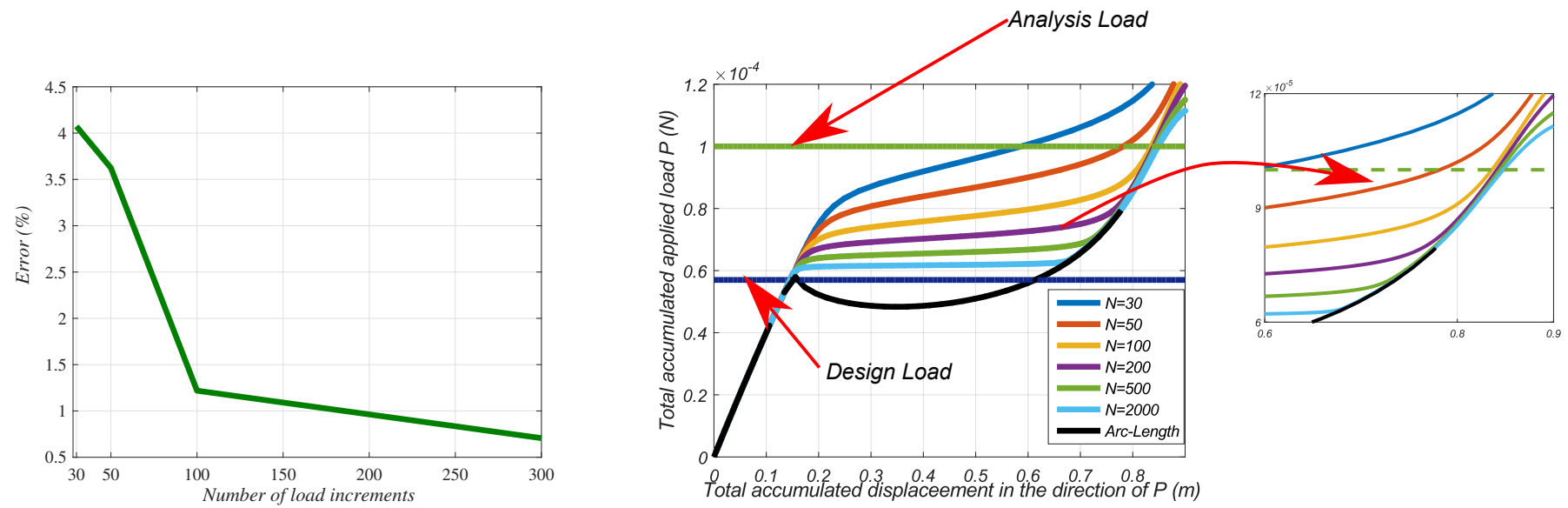

Figure 8: Numerical example 1. (a) Relative error of the vertical displacement at the application of the nodal load obtained by means of the incremental (Reg-TO) approach vs number of load increments for design in Figure $3_{f}$ for the corresponding design load (b) Variation of the post-buckling regime with the number of load increments for the design in Figure $3_{b}$.

It is important to emphasise that the post-buckling regime in Figure $8_{b}$ has been obtained for a final design, i.e. when densities are extremely close to either 1 or 0 . In this scenario, it is possible to obtain the true equilibrium path by means of the arc-length method. However, in the presence of intermediate densities, namely, at the initial and intermediate stages of the optimisation process, artificial instabilities pose a serious limitation for the robust application of the arc-length method, thus justifying the application of our stabilised approach. In this scenario, i.e. in the presence of intermediate densities, even the application of displacement control (application of Dirichlet boundary conditions instead of Neumann), does not preclude the development of artificial instabilities associated with these densities, hence, displacement control cannot be expected to yield the required robustness, specially in the context of very large deformations/displacements shown throughout Figure 8. 


\subsection{Numerical example 2}

The objective of this example is:

- O1 To test the performance of the Reg-TO stragey in the Cantilever Beam problem [11, 16], a classical problem in Topology Optimisation, but analysed in the context of extremely large deformations.

The geometry and boundary conditions are displayed in Figure 9 and the geometrical parameters, material properties, material parameters and simulation parameters are shown in Table 2.

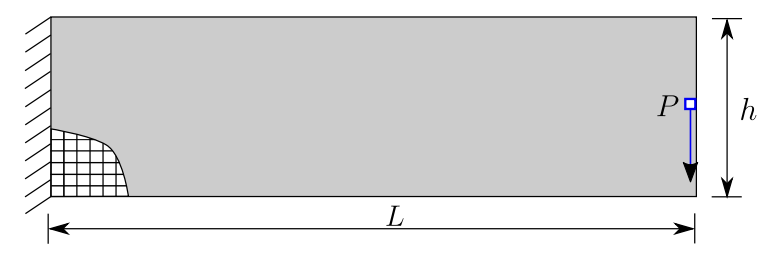

Figure 9: Numerical example 2. Geometry and boundary conditions.

Table 2: Numerical example 2. Material properties (see (7)), material parameters in (6) and simulation parameters.

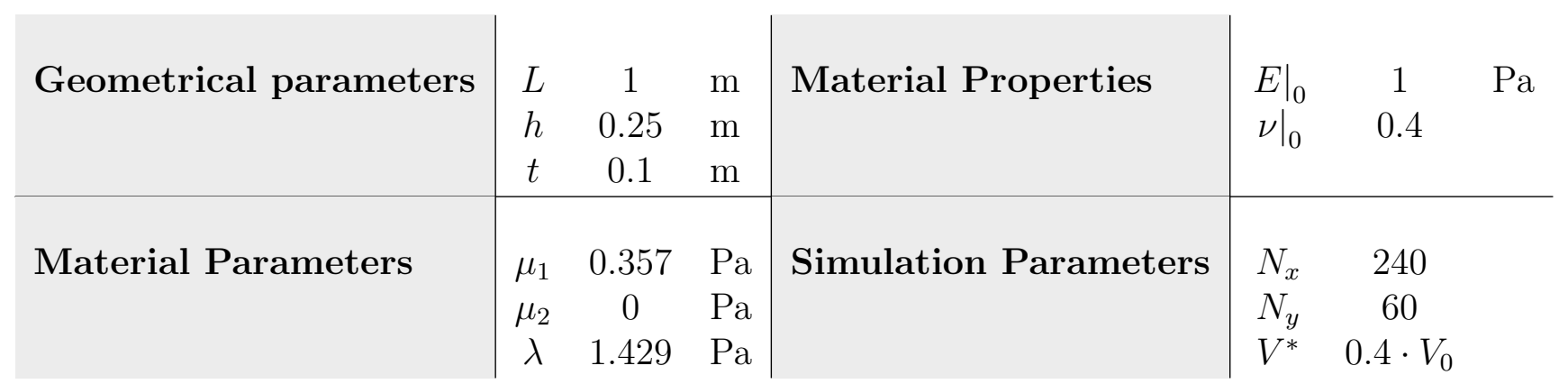

Figure 10 shows the designs obtained with Reg-TO for various design loads. The number of load increments was $N=30$ for all the cases. Figure 10 also displays the equilibrium paths associated with these designs. It is worth emphasising that the equilibrium paths have been obtained at a postprocessing level, using an arc-length and hence, ensuring that no artificial stiffness is introduced. In the equilibrium paths, the vertical axis displays the absolute value of the applied force $P$ (with the design load represented with a blue horizontal line) and the horizontal axis shows the absolute value of the displacement along the direction of the applied force and at its point of application. In all cases, the optimum designs do not exhibit limit points or softening prior to the design load, which is why a second stage TO phase by means of Hybrid-TO (see previous Section 5.1) has not been necessary.

Finally, Figure 11 shows the deformed configurations and contour plot distributions of the hydrostatic pressure $p$ (pressure in the sequel) for the optimum designs obtained. It is worth noticing how the evolution of the designs (for increasing values of the design load) leads to extremely simplified topologies at large displacements where the areas subjected to compressions tend to be minimised. 

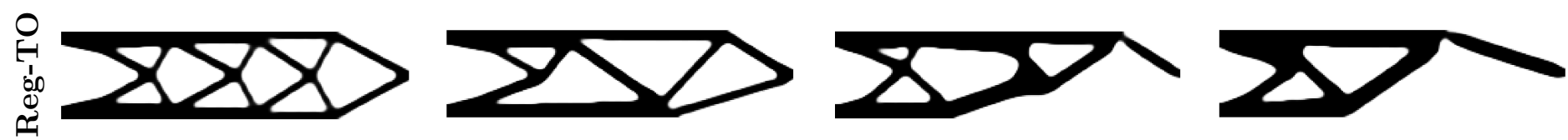

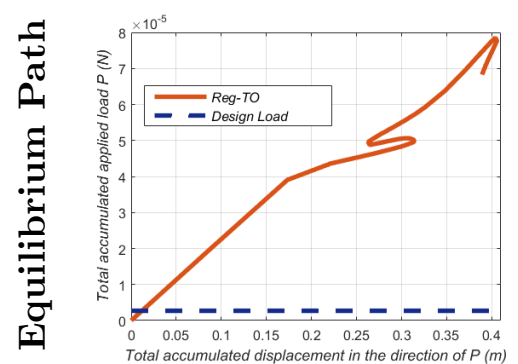

(a)
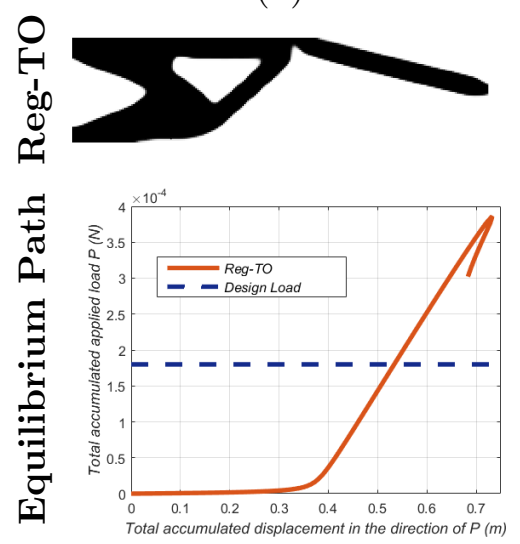

(e)

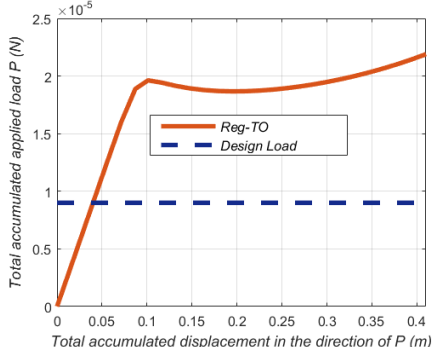

(b)
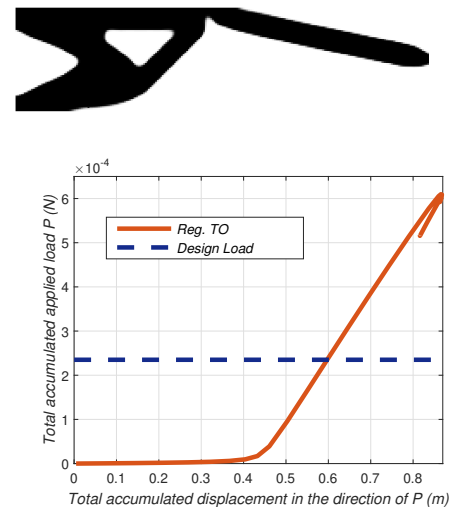

(f)

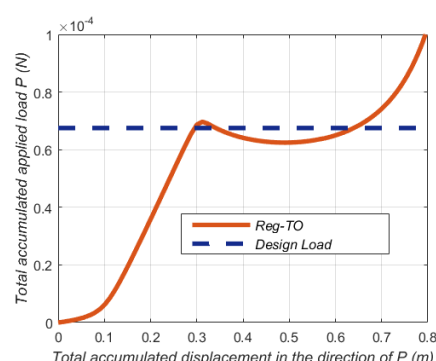

(c)
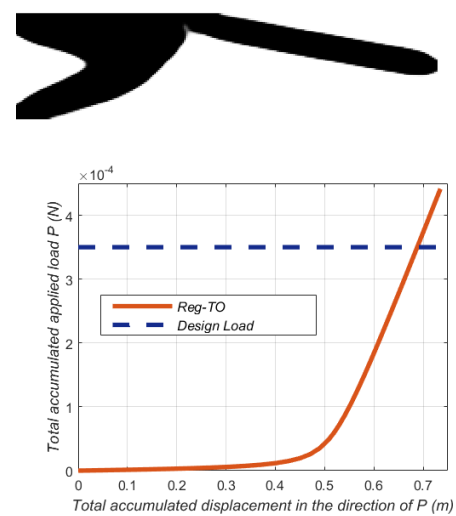

(g)

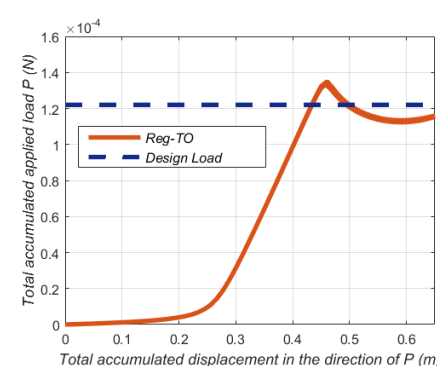

(d)
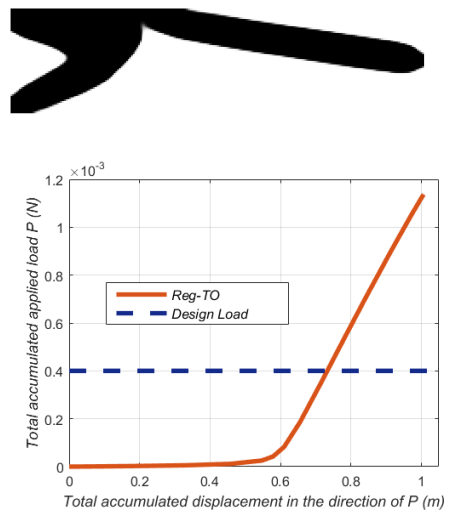

(h)

Figure 10: Numerical example 2. Optimal designs obtained with Reg-TO for loads: $P=\{0.27,0.9,6.7,12,18,23,35,40\} \times$ $10^{-5}$ and equilibrium paths obtained by means of an arc-length without extra stiffness.

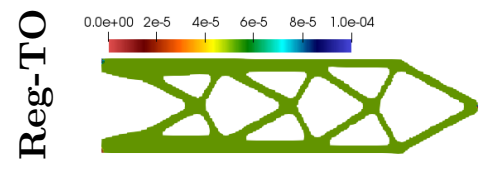

(a)

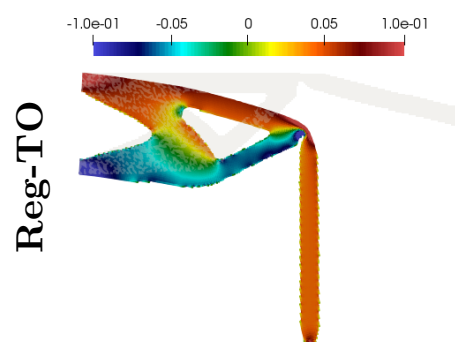

(e)

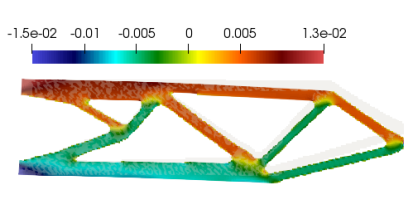

(b)

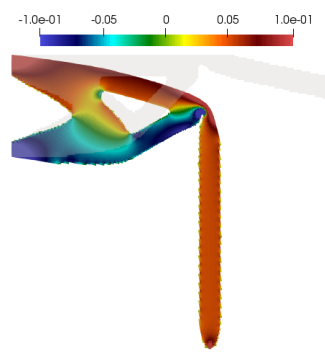

(f)

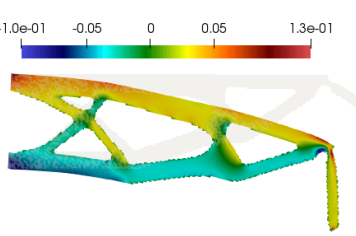

(c)

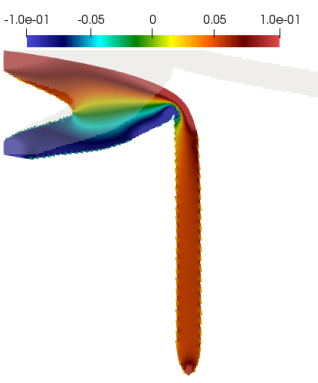

(g)

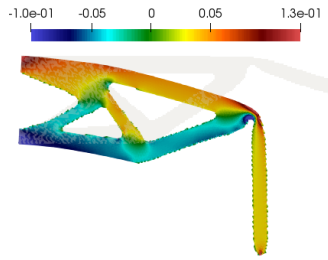

(d)

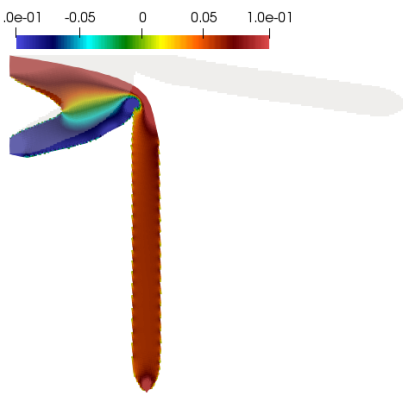

(h)

Figure 11: Numerical example 3. Contour plot distribution of the hydrostataic pressure $p$ associated with the designs obtained in Figure 10 for values of $P=\{0.27,0.9,6.7,12,18,23,35,40\} \times 10^{-5}$ (from left to right) with Reg-TO. 


\subsection{Numerical example 3}

The objective of this example is:

- O1 To test the performance of Reg-TO in another interesting problem in Topology Optimisation, named here as the two loads example [1].

The geometry and boundary conditions for this benchmark problem are displayed in Figure 12 and the geometrical parameters, material properties, material parameters and simulation parameters are shown in Table 3.

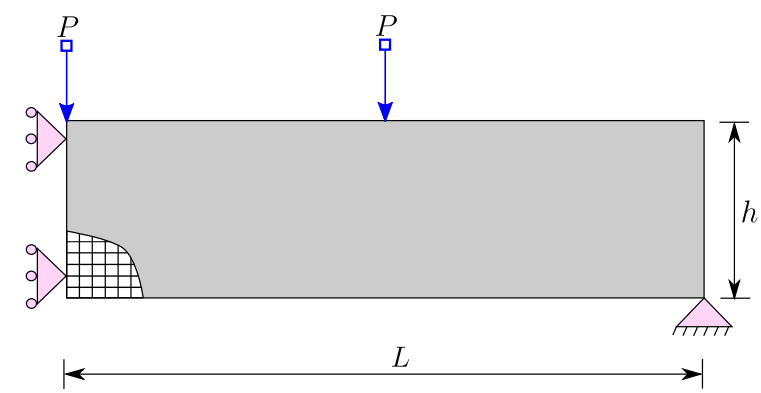

Figure 12: Numerical example 3. Geometry and boundary conditions.

Table 3: Numerical example 3. Material properties (see (7)), material parameters in (6) and simulation parameters.

\begin{tabular}{|c|c|c|c|c|c|c|}
\hline Geometrical parameters & $\begin{array}{l}L \\
h \\
t\end{array}$ & $\begin{array}{c}1 \\
0.25 \\
0.1 \\
\end{array}$ & $\begin{array}{l}\mathrm{m} \\
\mathrm{m} \\
\mathrm{m}\end{array}$ & Material Properties & $\begin{array}{c}\left.E\right|_{0} \\
\left.\nu\right|_{0}\end{array}$ & $\begin{array}{c}1 \\
0.4\end{array}$ \\
\hline Material Parameters & $\begin{array}{c}\mu_{1} \\
\mu_{2} \\
\lambda\end{array}$ & $\begin{array}{c}0.357 \\
0 \\
1.429\end{array}$ & $\begin{array}{l}\mathrm{Pa} \\
\mathrm{Pa} \\
\mathrm{Pa}\end{array}$ & Simulation Parameters & $\begin{array}{l}N_{x} \\
N_{y} \\
V^{*}\end{array}$ & $\begin{array}{c}240 \\
60 \\
0.4 \cdot V_{0}\end{array}$ \\
\hline
\end{tabular}

Figure 13 shows the designs obtained with Reg-TO for various design loads. The number of load increments was $N=30$ for all load cases. Figure 13 also displays the equilibrium paths associated with these designs, obtained in a similar manner to those in the previous examples. Clearly, the designs corresponding to figures $13_{d, e, g}$ exhibit a critical point prior to the design load. The reason for this is analogous to that previously explained in the numerical example in Section 5.1.

Using as initial guess these designs, we apply as in Section 5.1, the same continuation technique, where the Reg-TO stage is followed by a Hybrid-TO stage. Figure 14 compares the designs obtained after application of this continuation technique. Finally, Figure 15 shows the contour plot distributions of the hydrostatic pressure $p$ corresponding to the designs in Figure $13_{d, e, g}$, where those with critical points prior to the design load have been replaced with their counterparts in Figure 14. 

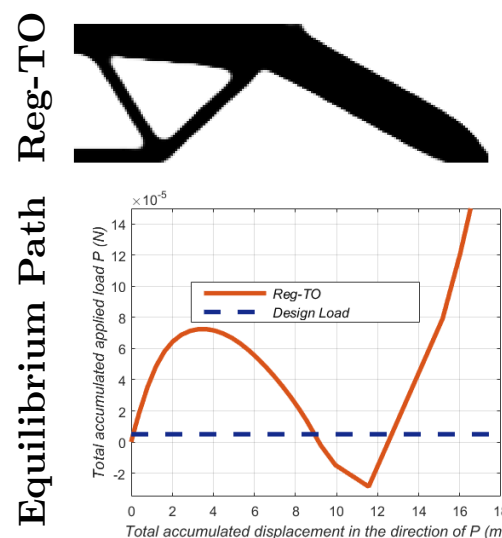

(a)
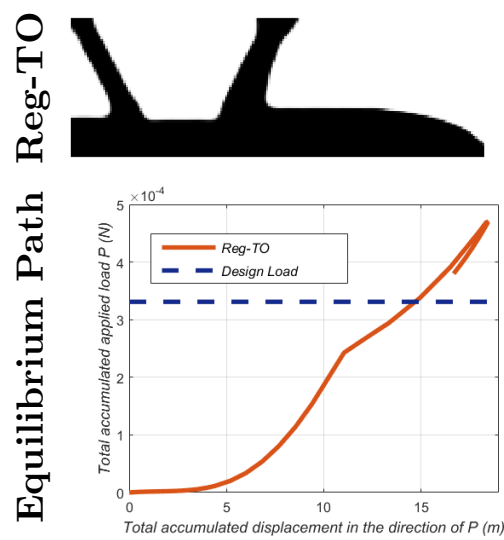

(e)
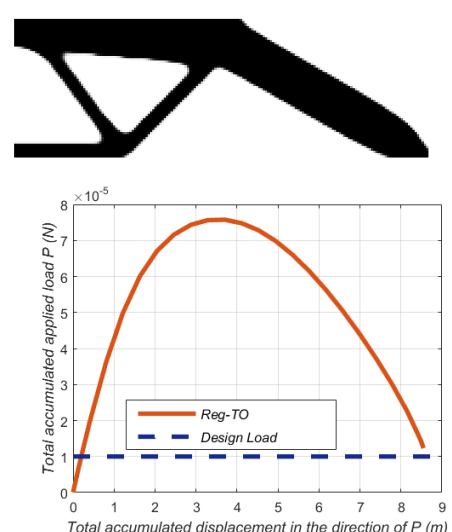

(b)
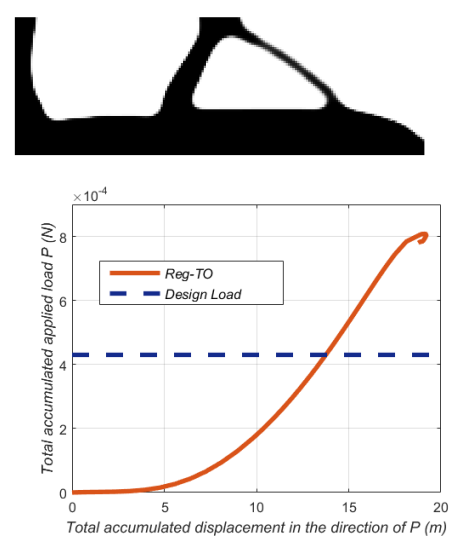

(f)
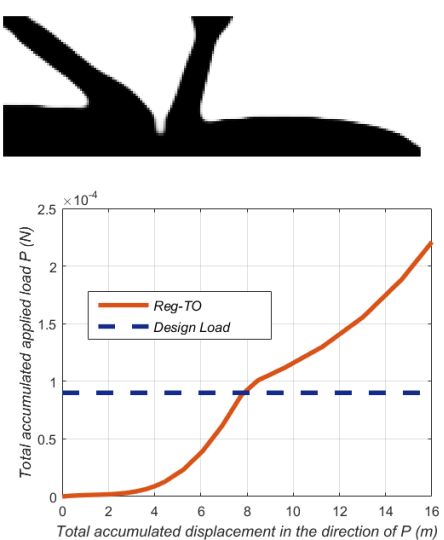

(c)
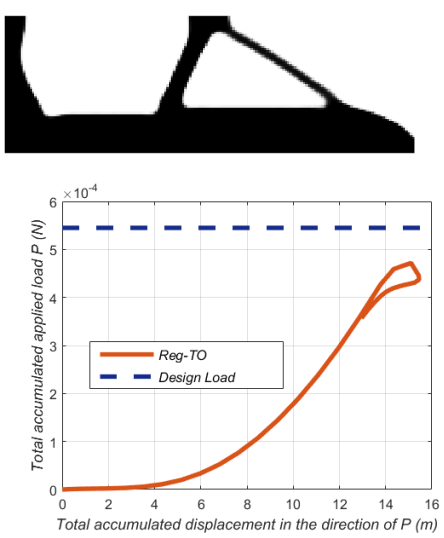

(g)
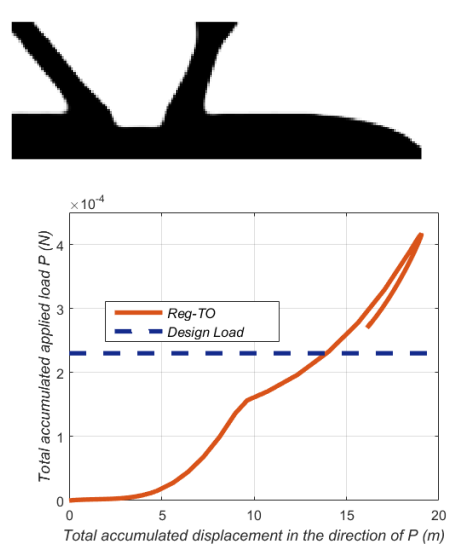

(d)
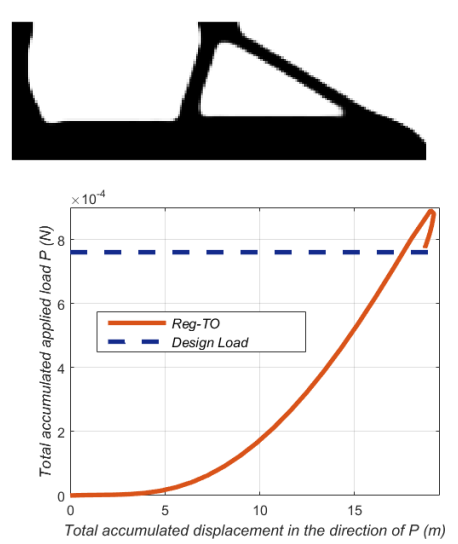

(h)

Figure 13: Numerical example 3. Optimal designs obtained by means of Reg-TO strategy for loads: $P=$ $\{0.5,1,9,23,33,43,54,76\} \times 10^{-5} \mathrm{~N}$. Equilibrium paths obtained by means of an arc-length without adding extra stiffness. 

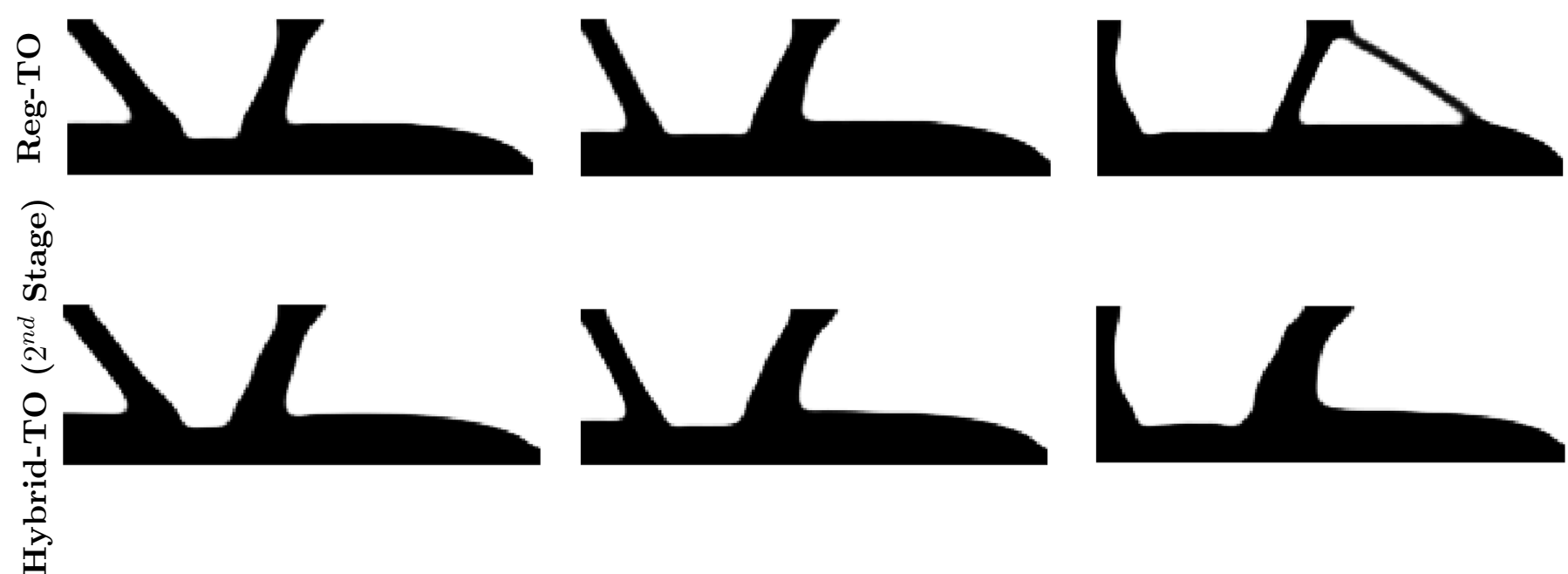

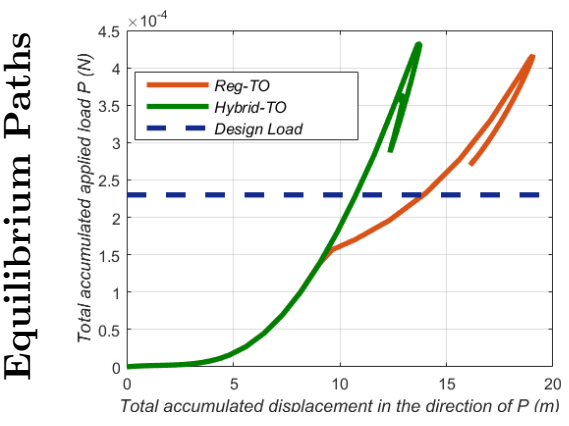

(a)

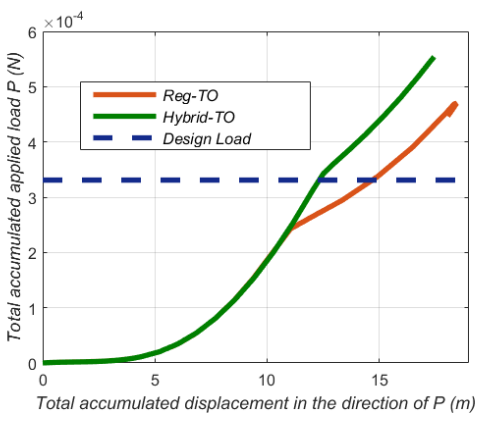

(b)

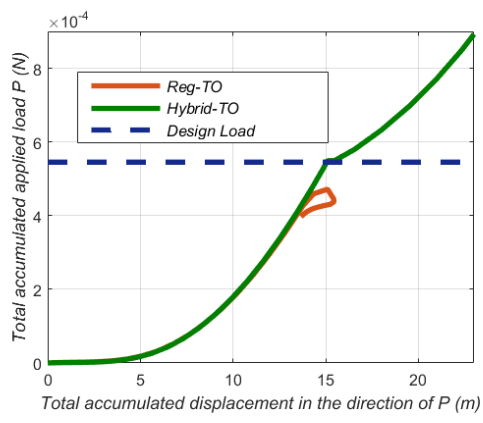

(c)

Figure 14: Numerical example 3. First row: designs obtained with Reg-TO. Second row: designs obtained by means of continuation method, namely, Reg-TO followed by Hybrid-TO. Third row: comparison of the performance of both designs. Cases corresponding to design loads $P=\{23,33,54\} \times 10^{-5} N$, namely, designs in Figures $13_{d}, 13_{e}$ and $13_{g}$, respectively. 


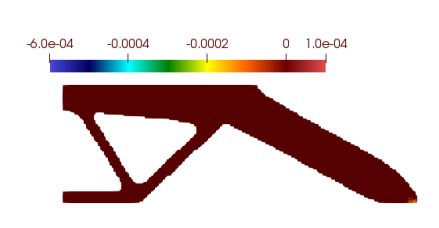

(a)

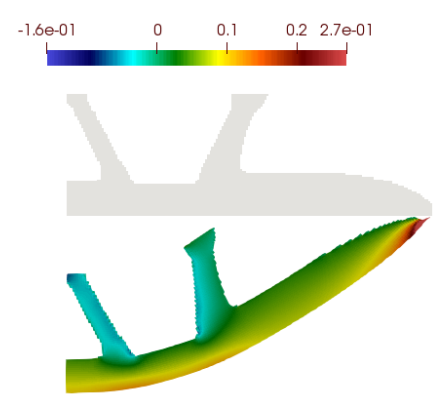

(e)

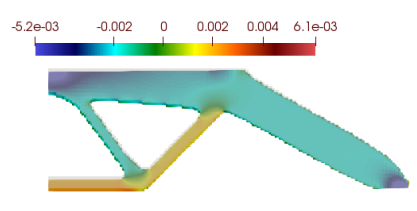

(b)

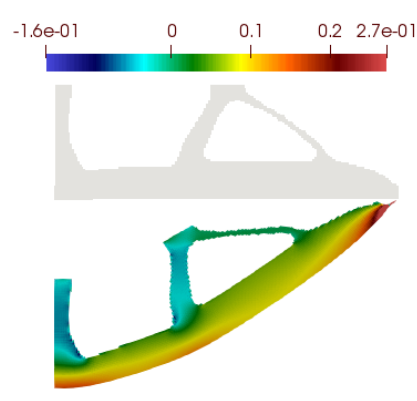

(f)

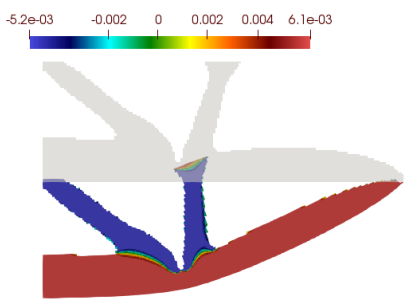

(c)

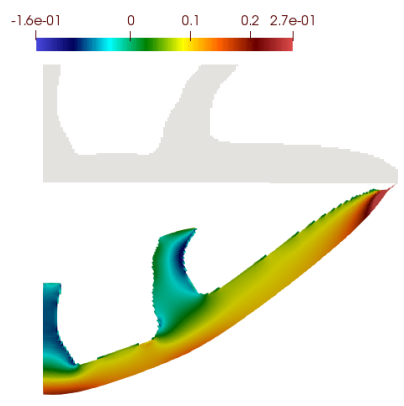

(g)

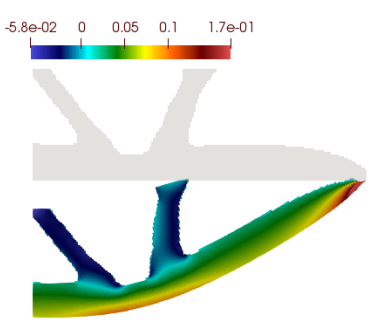

(d)

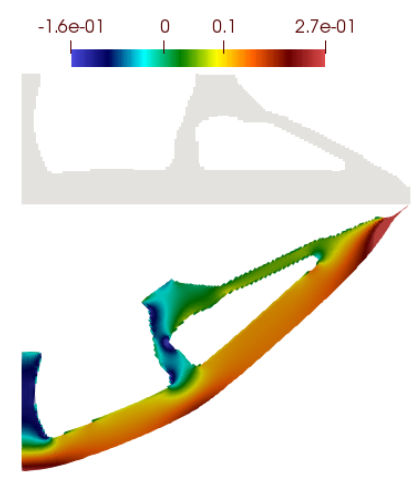

(h)

Figure 15: Numerical example 3. Contour plot distribution of the hydrostatic pressure $p$ associated with the designs obtained in Figure 13 for values of $P=\{0.5,1,9,23,33,43,54,76\} \times 10^{-5} N$ (from left to right), with Reg-TO and with continuation technique (Reg-TO followed by Hybrid-TO for (d), (e) and (g)). 


\subsection{Numerical example 4}

The objective of this example is:

- O1 To demonstrate the applicability and potential of the methodology described in Section 4 to the case of large scale three-dimensional problems.

The geometry and boundary conditions for this example are displayed in Figure 16 and the geometrical parameters, material properties, material parameters and simulation parameters are shown in Table 4.

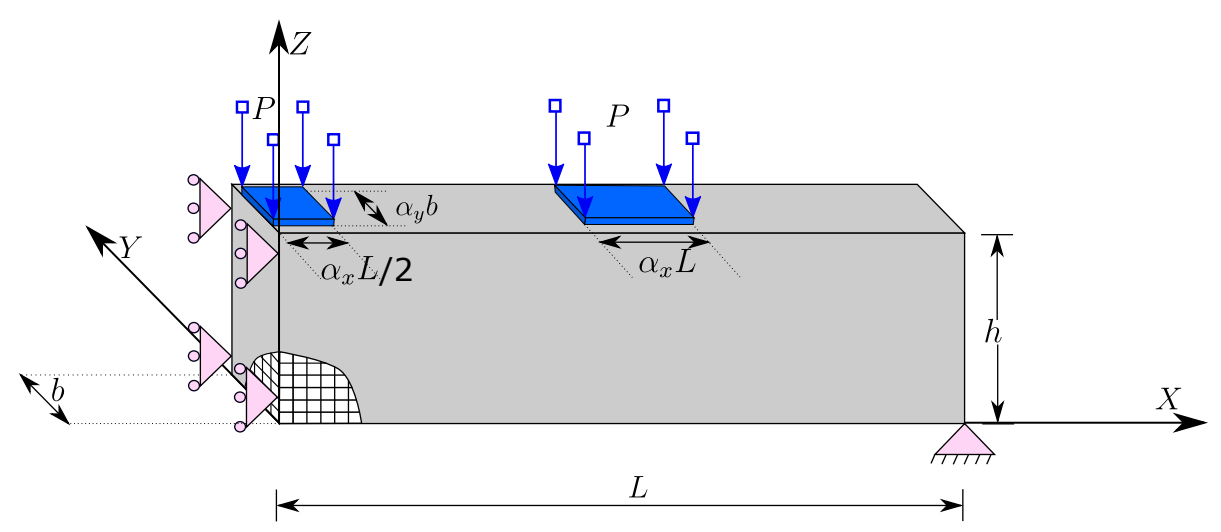

Figure 16: Numerical example 4. Geometry and boundary conditions. Only half of the domain in the $X$ direction represented (symmetric boundary conditions displayed at $X=0$ ), $\alpha_{x}=0.05$ and $\alpha_{y}=0.2$.

Table 4: Numerical example 4. Material properties (see (7)), material parameters in (6) and simulation parameters.

\begin{tabular}{|c|c|c|c|c|c|c|}
\hline Geometrical parameters & $\begin{array}{l}L \\
h \\
b\end{array}$ & $\begin{array}{c}1 \\
0.25 \\
0.1\end{array}$ & $\begin{array}{l}\mathrm{m} \\
\mathrm{m} \\
\mathrm{m}\end{array}$ & Material Properties & $\begin{array}{c}\left.E\right|_{0} \\
\left.\nu\right|_{0}\end{array}$ & $\begin{array}{c}1 \\
0.4\end{array}$ \\
\hline Material Parameters & $\begin{array}{c}\mu_{1} \\
\mu_{2} \\
\lambda\end{array}$ & $\begin{array}{c}0.357 \\
0 \\
1.429\end{array}$ & $\begin{array}{l}\mathrm{Pa} \\
\mathrm{Pa} \\
\mathrm{Pa}\end{array}$ & Simulation Parameters & $\begin{array}{l}N_{x} \\
N_{y} \\
N_{z} \\
V^{*}\end{array}$ & $\begin{array}{c}240 \\
60 \\
60 \\
0.4 \cdot V_{0}\end{array}$ \\
\hline
\end{tabular}

Figure 17 shows two zoomed-in details of the optimum design obtained with Reg-TO (employing $N=30$ load increments) and using a posteriori Hybrid-TO as a continuation technique. Finally, Figure 18 shows the contour plot distribution of the hydrostatic pressure $p$ for the optimum design. It is worth noticing how in this example, subjected to very large deformations and displacements, the final design manages to transfer the load from the compressed regions (in blue, associated with negative values of $p$ ) to the tractioned (in red, associated with positive values of $p$ ) horizontal slab. 

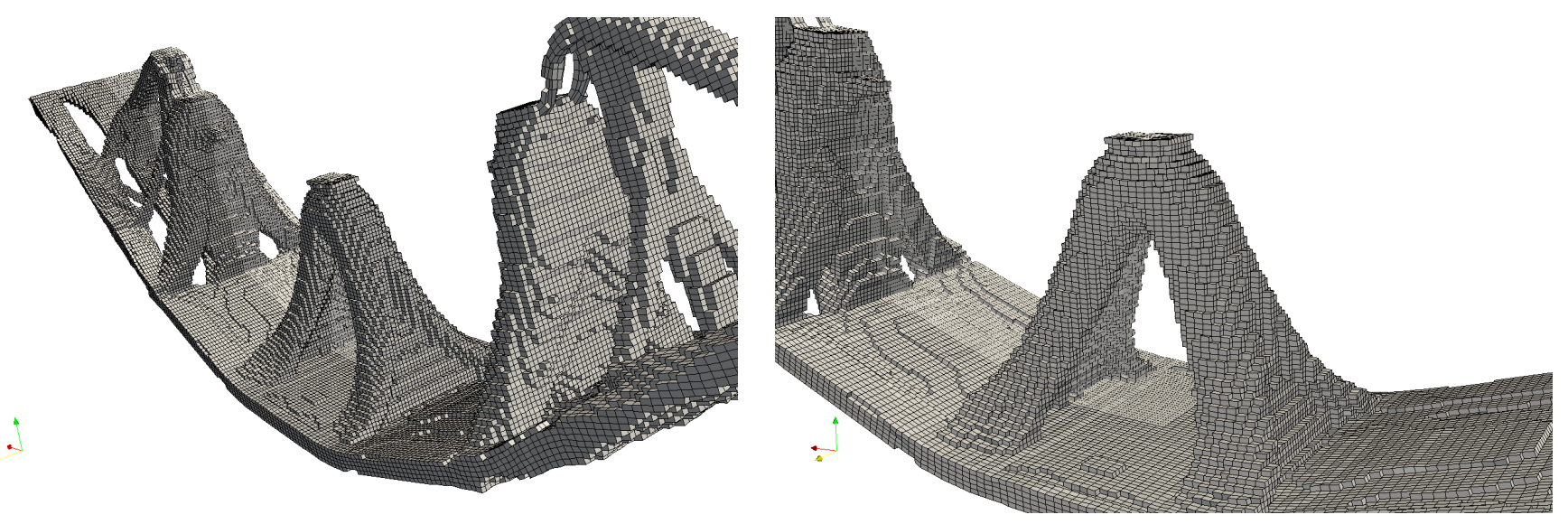

Figure 17: Numerical example 4. Optimum design zoomed details.

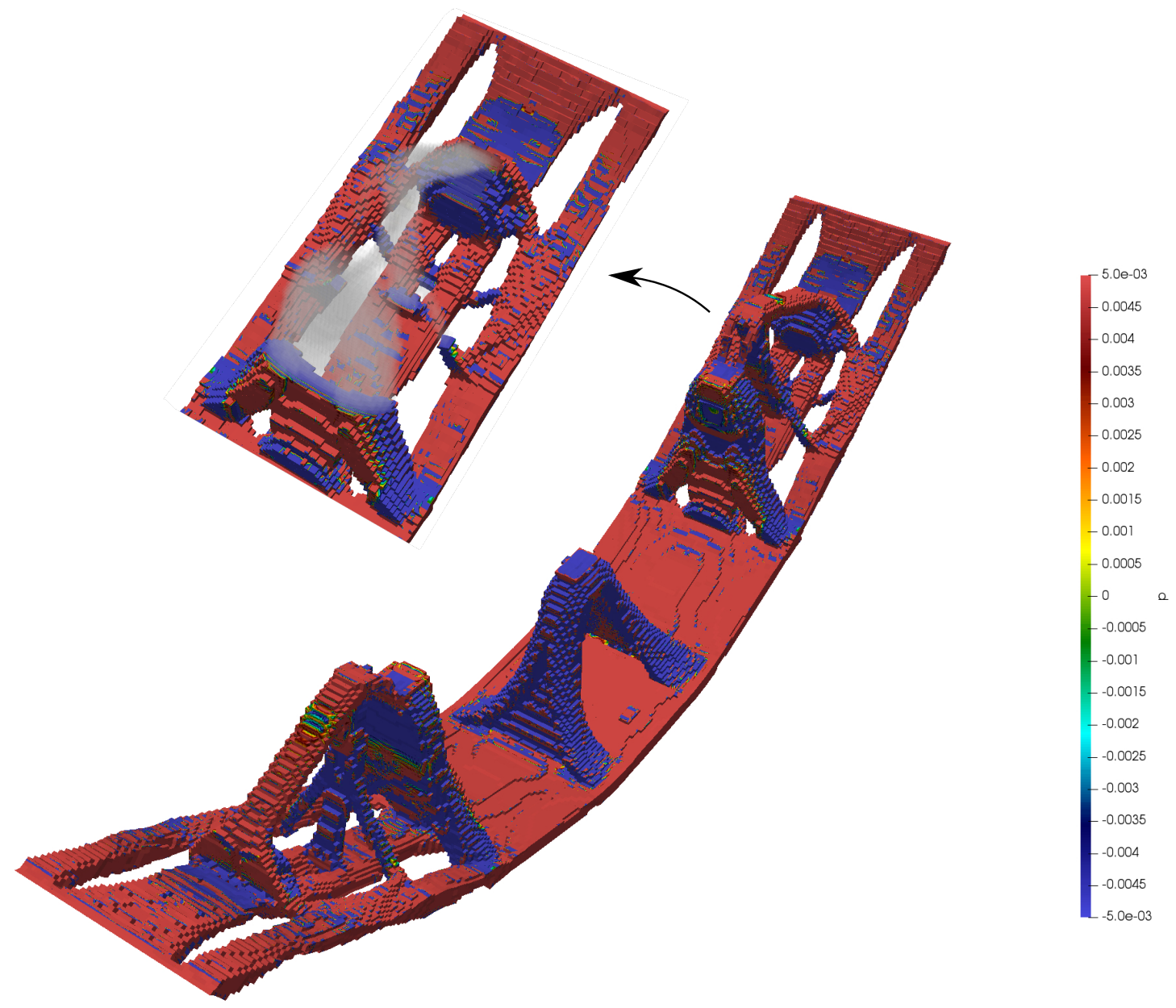

Figure 18: Numerical example 4. Contour plot distribution of hydrostatic pressure $p$. 


\section{Concluding remarks}

Inspired by the work in [1], this paper presents a computational approach for SIMP-based Topology Optimisation (TO) of hyperelastic materials at large strains. Through a series of challenging numerical examples, both in two and three dimensions, the paper demonstrates that the methodology put forward in [1], initially devised to alleviate numerical instabilities inherent to level-set TO, can be effectively modified and adapted to the case of SIMP-based TO. The methodology is shown to be successful at circumventing convergence difficulties due to the presence of intermediate density regions during intermediate TO design stages. The new computational approach seeks the relaxation of the original optimisation Lagrangian by combining the following two key features: (i) the equilibrium equations are solved approximately albeit in a consistently linearised incremental fashion, splitting the total design load in $N$ (user-defined) load increments; (ii) at each load increment, the elasticity tensor is locally regularised preventing its loss of positive definiteness and, hence, that of the tangent operator. Crucially, the performance of all the designs obtained in the numerical examples Section has been tested at a postprocessing stage without adding any source of artificial stiffness. Specifically, an arc-length NewtonRaphson method has been employed in conjunction with a ratio of the material parameters for void and solid regions of $10^{-12}$.

\section{Acknowledgements}

The first and third authors acknowledge the support provided by the Sêr Cymru National Research Network under the Ser Cymru II Fellowship Virtual engineering of the new generation of biomimetic artificial muscles, funded by the European Regional Development Fund. The first author also acknowledges the support provided by Ministerio de Ciencia, Innovación y Universidades, for the award of a Juan de la Cierva Formación Fellowship. The second, fourth and fifth authors acknowledge the support provided by the Ministerio de Economía, Industria y Competitividad through grant MTM2017-83740-P (Spain).

\section{References}

[1] R. Ortigosa, J. Martínez-Frutos, A. J. Gil, D. Herrero-Pérez, A new stabilisation approach for level-set based topology optimisation of hyperelastic materials, Structural and Multidisdiplinary Optimisation (2019) 1-29.

[2] M. P. Bendsøe, N. Kikuchi, Generating optimal topologies in structural design using a homogenization method, Computer Methods in Applied Mechanics and Engineering 71 (1988) 197-224.

[3] O. Sigmund, K. Maute, Topology optimization approaches a comparative review, Structural and Multidisdiplinary Optimisation 48 (2013) 1031-1055.

[4] N. Aage, E. Andreassen, B. Lazarov, O. Sigmund, Giga-voxel computational morphogenesis for structural design, Nature 550 (2017) 84-86.

[5] M. Zhou, G. Rozvany, The coc algorithm, part II: topological, geometrical and generalized shape optimization, Computer Methods in Applied Mechanics and Engineering 89 (1991) 309-336.

[6] G. Allaire, F. Jouve, T. A, Structural optimization using sensitivity analysis and a level-set method, Journal of Computational Physics 194 (2004) 363-393. 
[7] M. Wang, X. Wang, D. Guo, A level-set method for structural topology optimization, Computer Methods in Applied Mechanics and Engineering 192 (2003) 227-246.

[8] M. Burger, R. Stainko, Phase-field relaxation of topology optimization with local stress constraints, SIAM Journal on Control and Optimisation 192 (2003) 147-1466.

[9] J. Sokolowski, A. Zochowski, On the topological derivative in shape optimization, SIAM Journal on Control and Optimisation 37 (1999) 1251-1272.

[10] D. J. Munk, G. P. Steven, Topology and shape optimization methods using evolutionary algorithms: a review, Structural and Multidisciplinary Optimisation 52 (2015) 613-631.

[11] F. Wang, B. S. Lazarov, O. Sigmund, J. S. Jensen, Towards the stabilization of the low density elements in topology optimization with large deformation, Computer Methods in Applied Mechanics and Engineering 276 (2014) 453-472.

[12] F. Chen, Y. Wang, M. Wang, Y. Zhang, Topology optimization of hyperelastic structures using a level set method, Journal of Computational Physics 351 (2017) 437-454.

[13] J. Bonet, A. J. Gil, R. D. Wood, Nonlinear solid mechanics for finite element analysis: statics, Cambridge University Press, Cambridge, 2016.

[14] M. P. Bendsøe, O. Sigmund, Topology optimization, Springer-Verlag, Berlin, 2003. Theory, methods and applications.

[15] F. Wang, B. Lazarov, O. Sigmund, J. Jensen, Interpolation scheme for fictitious domain techniques and topology optimization of finite strain elastic problems, Computer Methods in Applied Mechanics and Engineering 276 (2014) 453-472.

[16] R. Lahuerta, E. Simoes, E. Campello, P. Pimenta, E. Silva, Towards the stabilization of the low density elements in topology optimization with large deformation, Computational Mechanics 52 (2013) 779-797.

[17] P. G. Ciarlet, Mathematical Elasticity. Volume 1: Three Dimensional Elasticity, Elsevier, 1988.

[18] J. Marsden, T. Hughes, Mathematical Foundations of Elasticity, Dover Civil and Mechanical Engineering Series, Dover, 1994.

[19] T. J. R. Hughes, A variational approach for materially stable anisotropic hyperelasticity, International Journal of Solids and Structures 42 (2005) 4352-4371.

[20] J. M. Ball, Convexity conditions and existence theorems in nonlinear elasticity, Archive for Rational Mechanics and Analysis 63 (1976) 337-403.

[21] P. N. J. Schröder, Invariant formulation of hyperelastic transverse isotropy based on polyconvex free energy functions, International Journal of Solids and Structures 40 (2003) 401-445.

[22] J. M. Ball, Some open problems in elasticity, in: Geometry, mechanics, and dynamics, Springer, New York, 2002, pp. 3-59. 
[23] R. Ortigosa, A. J. Gil, C. Hesch, A computational framework for polyconvex large strain elasticity for geometrically exact beam theory, Computer Methods in Applied Mechanics and Engineering 317 (2015) 277-303.

[24] A. Klarbring, N. Strömberg, Topology optimization of hyperelastic bodies including non-zero prescribed displacements, Structural and Multidisciplinary Optimization 47 (2013) 37-48.

[25] Y. Luo, M. Wang, Z. Kang, Topology optimization of geometrically nonlinear structures based on an additive hyperelasticity technique, Computer Methods in Applied Mechanics and Engineering 286 (2015) 422-441.

[26] Y. Luo, M. Li, Z. Kang, Topology optimization of hyperelastic structures with frictionless contact supports, International Journal of Solids and Structures 81 (2016) 373-382.

[27] R. Lahuerta, E. Simões, E. Campello, P. Pimenta, E. Silva, Towards the stabilization of the low density elements in topology optimization with large deformation, Computational Mechanics 52 (2013) 779-797.

[28] M. Wallin, M. Ristinmaa, Finite strain topology optimization based on phase-field regularization, Structural and Multidisciplinary Optimization 51 (2015) 305-317.

[29] K. James, H. Waisman, Layout design of a bi-stable cardiovascular stent using topology optimization, Computer Methods in Applied Mechanics and Engineering 305 (2016) 869-890.

[30] T. Buhl, C. Pedersen, O. Sigmund, Stiffness design of geometrically nonlinear structures using topology optimization, Structural and Multidisciplinary Optimization 19 (2000) 93-104.

[31] B. Lazarov, M. Schevenels, O. Sigmund, Robust design of large-displacement compliant mechanisms, Mechanical Sciences 2 (2011) 175-182.

[32] Q. Xia, T. Shi, Stiffness optimization of geometrically nonlinear structures and the level set based solution, International Journal for Simulation and Multidisciplinary Design Optimization 7 (2016) A3.

[33] L. Liu, J. Xing, Q. Yang, Y. Luo, Design of large-displacement compliant mechanisms by topology optimization incorporating modified additive hyperelasticity technique, Mathematical Problems in Engineering (2017).

[34] H. Chung, O. Amir, H. A. Kim, Level-set topology optimization considering nonlinear thermoelasticity, arXiv, 1909.11185 (2019).

[35] J. Bonet, A. J. Gil, R. Ortigosa, On a tensor cross product based formulation of large strain solid mechanics, International Journal of Solids and Structures 84 (2016) 49-63.

[36] J. Bonet, A. J. Gil, R. Ortigosa, A computational framework for polyconvex large strain elasticity, Comput. Methods Appl. Mech. Engrg. 283 (2015) 1061-1094.

[37] F. Wang, B. S. Lazarov, O. Sigmund, On projection methods, convergence and robust formulations in topology optimization, Structural and Multidisciplinary Optimization 43 (2011) 767-784. 
[38] M. P. Bendsøe, O. Sigmund, Material interpolation schemes in topology optimization, Archives of Applied Mechanics 69 (1999) 635-654.

[39] K. Svanberg, The method of moving asymptotesa new method for structural optimization, International Journal for Numerical Methods in Engineering 24 (1987) 359-373.

[40] A. Limkilde, A. Evgrafov, J. Gravesen, On reducing computational effort in topology optimization: we can go at least this far!, Struct. Multidiscip. Optim. 58 (2018) 2481-2492.

[41] F. Ferrari, O. Sigmund, Revisiting topology optimisation with buckling constraints, Structural and Multidisciplinary Optimisation 59 (2019) 1401-1415.

[42] J. Bonet, R. D. Wood, Nonlinear Continuum Mechanics for Finite Element Analysis, Cambridge University Press, second edition, 2008. 